\title{
Exotic Nonlinear Analysis: Part 3: Yang-Mills Quantum Fields
}

\author{
Jean-Bernard Pécot \\ Communicated by Y. Charles Li, received August 30, 2006.
}

\begin{abstract}
We generalize the inverse function theorem to show the existence of an infinity of smooth classical symmetric Yang-Mills gauge fields and of some particular kinds of super-symmetric Yang-Mills gauge fields in a smooth 3-dimensional compact manifold with boundary for any finite interval of time or in whole $\mathbb{R}^{4}$.
\end{abstract}

\section{Contents}

1. Introduction 303

2. A generalization of the inverse function theorem 304

3. Application to the Yang-Mills equation 307

4. Strength tensors of a certain kind of super-symmetry type 329

5. Conclusion 333

References $\quad 334$

\section{Introduction}

This paper is the third and last one of an attempt to improve global inversion in nonlinear Analysis. In a first part, we go to the roots of the inverse function theorem back. We generalize this inverse function theorem to obtain precise bounds for the rays of the bowls in which the inversion occurs. Our main reference is Avez's treatise [1].

In a second part, we apply this topological result to prove the existence of an infinity of smooth symmetric gauge fields with commutators for one Lie algebra in a smooth compact manifold with boundary of $\mathbb{R}^{3}$ for a finite interval of time or in whole $\mathbb{R}^{4}$. This one Lie algebra model covers the classical interactions: on the first hand, the electromagnetic and the weak theories for the $U(1)$ gauge subgroup

1991 Mathematics Subject Classification. 46, 81,35.

Key words and phrases. Inverse function theorem, Yang-Mills gauge fields.

(C)2007 International Press 
diagonally embedded in $S U(2) \times U(1)$ and on the other hand the nuclear strong forces with the gauge group $S U(3)$.

In a third part, we apply our generalization of the inverse function theorem to prove the existence of some classes of super-symmetric Yang-Mills fields, still in a smooth compact manifold with boundary of $\mathbb{R}^{3}$ for a finite interval of time or in whole $\mathbb{R}^{4}$.

The main analytical tool of this paper is a transformation of a system of linear wave equations derived at any finite order into a first order system of large rank; inspired by Michael Taylor books [5], we will use the so-called method of the "finite propagation speed" in a thin version that we will call "tesseralization" to show the existence and the uniqueness of the solution of this "huge" first order system.

Since hyperbolic problems (in the linear case or not) are more tricky than the parabolic ones, we will have to use finest computations than in the couple of our first papers but for less precise results: indeed, we won't obtain the uniqueness of the solution but only the existence.

Three other references have guided us: Claude Itzykson's and Jean-Bernard Zuber's book [3], Theodore Frankel's book [2] and the three volumes of "The Quantum Theory of Fields" by Steven Weinberg [6].

\section{A generalization of the inverse function theorem}

2.1. Statement of the theorem. We denote by $B_{S}(c, r)$ the open ball of ray $r$, centered at $c$ in a norm space $S$ :

$$
B_{S}(c, r)=\left\{x \in S,\|x-c\|_{S}<r\right\} .
$$

TheOREM 2.1. 1) Consider a sequence of Banach spaces $\left(E_{n}\right)_{n \geq 0}$ with continuous inclusions:

$$
\ldots E_{n+1} \subset E_{n} \subset E_{n-1} \subset \cdots \subset E_{0}
$$

with:

$$
\forall n \geq 0, \quad\|x\|_{E_{n+1}} \geq\|x\|_{E_{n}} .
$$

2) $f$ is an operator in $C^{0}\left(E_{n+2}, \mathcal{F}_{n}\right)$, for any $n \geq 0$, where $\left(\mathcal{F}_{n}\right)_{n \geq 0}$ is a sequence of Banach spaces with continuous inclusions:

$$
\ldots \mathcal{F}_{n+1} \subset \mathcal{F}_{n} \subset \mathcal{F}_{n-1} \subset \ldots \subset \mathcal{F}_{0}
$$

and for any $x, y \in E_{n+2}$, the difference $f(x)-f(y) \in F_{n}$, where $\left(F_{n}\right)_{n \geq 0}$ is another sequence of Banach spaces with:

$$
\left\{\begin{array}{l}
F_{n} \subset \mathcal{F}_{n}, \quad n \geq 0 \text { (with a continuous injection) } \\
\|\cdot\|_{\mathcal{F}_{n}}=\|\cdot\|_{F_{n}}
\end{array} .\right.
$$

Suppose that $D f(x)$ for any $x \in E_{n+2}, n \geq 0$, exists in $L\left(E_{n+2}, F_{n}\right)$ and that $x \rightarrow D f(x)$ is in $C^{0}\left(E_{n+2}, L\left(E_{n+2}, F_{n}\right)\right)$.

3) Consider a sequence $\left(A_{n}\right)_{n \geq 0}$ of sets with:

a) $\forall n \geq 0, m \geq 0, A_{n} \subset E_{m}$

b) $\forall a_{n} \in A_{n}$ :

$$
\forall m \geq 0, \quad\left\|a_{n}\right\|_{E_{m}} \leq\left\|a_{n}\right\|_{E_{n}} .
$$

4) For any $a_{m} \subset A_{m},\left[D f\left(a_{m}\right)\right]^{-1}$ exists in $L\left(F_{n}, E_{n+1}\right)$ for any $n \geq 0$, with:

$$
\left\|\left[D f\left(a_{m}\right)\right]^{-1}\right\|_{L\left(F_{n}, E_{n+1}\right)} \leq \alpha_{m}\left\|a_{m}\right\|_{E_{m}}^{r}+\beta_{m},
$$


for a real $r$ independent of $m$ and of $n$, where $\alpha_{m}$ and $\beta_{m}$ are positive numbers only depending on $m$ and so independent of $n$.

5) There exists a positive real $\gamma$ independent of $n$ such that for any couple $(x, y)$ in $E_{n+2} \times E_{n+2}$ :

$\|D f(x)-D f(y)\|_{L\left(E_{n+2}, F_{n}\right)} \leq \gamma\left(\|x\|_{E_{n+2}}+\|y\|_{E_{n+2}}+1\right)\|x-y\|_{E_{n+2}}$.

6) For any $m \geq 0$ :

$$
12 \beta_{m} \gamma>1 \text {. }
$$

7) For any $n \geq 0$ and $(x, y) \in E_{n+1}^{2}$ :

$$
\|x-y\|_{E_{n+1}} \leq c_{n}(x, y)\|f(x)-f(y)\|_{F_{n}}
$$

where $c_{n}$ is a continuous operator from $E_{n+1} \times E_{n+1}$ into $\mathbb{R}^{+}$.

8) $x \rightarrow f(x)-f(0)$ acts from $A_{m}$ into $B_{\alpha(m)}$, where $\alpha$ is a function from $\mathbb{N}$ into $\mathbb{N}$ and $\left(B_{m}\right)_{m>0}$ a sequence of sets such that:

a) $\forall n, m \geq 0, \quad B_{n} \subset F_{m}$

b) $\forall b_{n} \in B_{n}$ :

$$
\forall m \geq 0, \quad\left\|b_{n}\right\|_{F_{m}} \leq\left\|b_{n}\right\|_{F_{n}} .
$$

9) We consider a sequence of sets $\left(C_{n}\right)_{n \geq 0}$ with:

a) $\forall n, m \geq 0, \quad C_{n} \subset F_{m}$

b) $\forall c_{n} \in C_{n}$ :

$$
\forall m \geq 0, \quad\left\|c_{n}\right\|_{F_{m}} \leq\left\|c_{n}\right\|_{F_{n}} .
$$

c) $\bigcup_{n \geq 0} C_{n}$ is dense in $F_{m}$, for any $m \geq 0$.

Then, for any $a_{m}$ in $A_{m}$ (this for any $m \geq 0$ ) and for any $n \geq 0, f^{-1}$ exists from $B_{F_{n}}\left(f\left(a_{m}\right), \frac{r_{m}}{\alpha_{m}\left\|a_{m}\right\|_{E_{m}}^{r}+\beta_{m}}\right)$ into $B_{E_{n+1}}\left(a_{m}, 2 r_{m}\right)$, for any $r_{m}$ such that:

$$
r_{m}<\frac{1}{4\left(\alpha_{m}\left\|a_{m}\right\|_{E_{m}}^{r}+\beta_{m}\right) \times \gamma\left(2\left\|a_{m}\right\|_{E_{m}}+3\right)} .
$$

2.2. Proof of Theorem 2.1. Consider $a_{m}$ in $A_{m}$ and the operator $g$ defined by:

$$
g(x)=x-\left[D f\left(a_{m}\right)\right]^{-1}\left(f(x)-f\left(a_{m}\right)\right) .
$$

According to assumptions 2) and 4$), g$ is in $C^{1}\left(E_{n+2}, E_{n+1}\right)$ for any $n \geq 0$ and:

$$
\left\{\begin{array}{l}
g\left(a_{m}\right)=a_{m} \\
D g(x)=\left[D f\left(a_{m}\right)\right]^{-1}\left[D f\left(a_{m}\right)-D f(x)\right]
\end{array},\right.
$$

for any $x$ in $E_{n+2}$ (since $x \in E_{n+2}, x \in E_{n+1}$ so that:

$$
\left.x=\left[D f\left(a_{m}\right)\right]^{-1}\left[D f\left(a_{m}\right) x\right] .\right)
$$

We suppose that $x \in B_{E_{n+2}}\left(a_{m}, 2 r_{m}^{\prime}\right)$, with:

$$
r_{m}^{\prime}<r_{m}
$$

where $r_{m}$ satisfies (1). So, according to assumption 6$), r_{m}<1$. We get, according to assumptions 4), 5) and $3 \mathrm{~b})$ :

$\|D g(x)\|_{L\left(E_{n+2}, E_{n+1}\right)} \leq\left(\alpha_{m}\left\|a_{m}\right\|_{E_{m}}^{r}+\beta_{m}\right) \times \gamma\left(2\left\|a_{m}\right\|_{E_{m}}+3\right) \times 2 r_{m}^{\prime}$.

Therefore, according to (1):

$$
\|D g(x)\|_{L\left(E_{n+2}, E_{n+1}\right)} \leq \frac{1}{2} .
$$

So, we obtain for any $x \in B_{E_{n+2}}\left(a_{m}, 2 r_{m}^{\prime}\right)$ : 


$$
\left\|g(x)-g\left(a_{m}\right)\right\|_{E_{n+1}} \leq \frac{1}{2}\left\|x-a_{m}\right\|_{E_{n+2}}<r_{m}^{\prime} .
$$

Since $g\left(a_{m}\right)=a_{m}$, we obtain that:

$$
g\left[B_{E_{n+2}}\left(a_{m}, 2 r_{m}^{\prime}\right)\right] \subset B_{E_{n+1}}\left(a_{m}, r_{m}^{\prime}\right) .
$$

For $y_{p} \in B_{F_{n}}\left(f\left(a_{m}\right)-f(0), \frac{r_{m}}{\alpha_{m}\left\|a_{m}\right\|_{E_{m}}^{r}+\beta_{m}}\right) \cap C_{p}$, we consider the operator $h_{y_{p}}$ defined by:

$$
\begin{aligned}
& h_{y_{p}}(x)=x-\left[D f\left(a_{m}\right)\right]^{-1}\left(f(x)-f(0)-y_{p}\right) \\
& h_{y_{p}}(x)=g(x)-\left[D f\left(a_{m}\right)\right]^{-1}\left(f\left(a_{m}\right)-f(0)-y_{p}\right) .
\end{aligned}
$$

Let's show that $h_{y_{p}}$ applies $B_{E_{n+2}}\left(a_{m}, 2 r_{m}^{\prime}\right)$ into $B_{E_{n+1}}\left(a_{m}, 2 r_{m}^{\prime}\right)$ for any $n \geq 0$, where $r_{m}^{\prime}$ is such that:

We get:

$$
y_{p} \in B_{F_{n}}\left(f\left(a_{m}\right)-f(0), \frac{r_{m}^{\prime}}{\alpha_{m}\left\|a_{m}\right\|_{E_{m}}^{r}+\beta_{m}}\right) .
$$

$$
\begin{aligned}
& \left\|h_{y_{p}}(x)-a_{m}\right\|_{E_{n+1}} \leq\left\|g(x)-a_{m}\right\|_{E_{n+1}}+\left\|\left[D f\left(a_{m}\right)\right]^{-1}\right\|_{L\left(F_{n}, E_{n+1}\right)} \times \\
& \left\|f\left(a_{m}\right)-f(0)-y_{p}\right\|_{F_{n}} \\
& \left\|h_{y_{p}}(x)-a_{m}\right\|_{E_{n+1}}<r_{m}^{\prime}+\left(\alpha_{m}\left\|a_{m}\right\|_{E_{m}}^{r}+\beta_{m}\right) \times \frac{r_{m}^{\prime}}{\alpha_{m}\left\|a_{m}\right\|_{E_{m}}^{r}+\beta_{m}} \\
& \left\|h_{y_{p}}(x)-a_{m}\right\|_{E_{n+1}}<2 r_{m}^{\prime} .
\end{aligned}
$$

So, $h_{y_{p}}$ applies $B_{E_{n+2}}\left(a_{m}, 2 r_{m}\right)$ into $B_{E_{n+1}}\left(a_{m}, 2 r_{m}\right)$ for any $n \geq 0$. Moreover, for $x, y \in B_{E_{n+2}}\left(a_{m}, 2 r_{m}\right)$ :

$$
\begin{aligned}
\left\|h_{y_{p}}(x)-h_{y_{p}}(y)\right\|_{E_{n+1}} & \leq\|g(x)-g(y)\|_{E_{n+1}} \\
\left\|h_{y_{p}}(x)-h_{y_{p}}(y)\right\|_{E_{n+1}} & \leq \frac{1}{2}\|x-y\|_{E_{n+2} .} .
\end{aligned}
$$

Now, we consider the following sequence $\left(z_{N}\right)_{N \geq 0}$ :

We get:

$$
\left\{\begin{array}{l}
z_{0}=a_{m} \\
z_{N}=h_{y_{p}}\left(z_{N-1}\right), \quad N \geq 1
\end{array} .\right.
$$

$$
\left\|z_{N+p}-z_{N}\right\|_{E_{n}} \leq \sum_{k=0}^{p-1}\left\|z_{N+p-k}-z_{N+p-k-1}\right\|_{E_{n}} .
$$

By rough majorations, for $N \geq 2$ :

$$
\begin{aligned}
&\left\|z_{N+p}-z_{N}\right\|_{E_{n}} \leq \sum_{k=0}^{p-1}\left(\frac{1}{2}\right)^{p-k-1}\left\|z_{N+1}-z_{N}\right\|_{E_{p+n}} \\
&\left\|z_{N+p}-z_{N}\right\|_{E_{n}} \leq\left(\frac{1}{2}\right)^{N} \times 2 \times\left\|z_{1}-z_{0}\right\|_{E_{p+n+N}} \\
&\left\|z_{N+p}-z_{N}\right\|_{E_{n}} \leq\left(\frac{1}{2}\right)^{N-2} \times\left\|h_{y_{p}}\left(a_{m}\right)-a_{m}\right\|_{E_{p+n+N}} \\
&\left\|z_{N+p}-z_{N}\right\|_{E_{n}} \leq\left(\frac{1}{2}\right)^{N-2} \times\left\|\left[D f\left(a_{m}\right)\right]^{-1}\right\|_{L\left(F_{p+n+N-1} ; E_{p+n+N}\right)} \times \\
&\left\|f\left(a_{m}\right)-f(0)-y_{p}\right\|_{F_{p+n+N-1}} .
\end{aligned}
$$


Since $y_{p} \in C_{p}$ and, by assumption 8), $f\left(a_{m}\right)-f(0) \in B_{\alpha(m)}$, according to assumption 9) and assumption 8):

$$
\begin{aligned}
& \left\|z_{N+p}-z_{N}\right\|_{E_{n}} \leq\left(\frac{1}{2}\right)^{N-2} \times\left(\alpha_{m}\left\|a_{m}\right\|_{E_{m}}^{r}+\beta_{m}\right) \\
& \times\left[\left\|f\left(a_{m}\right)-f(0)\right\|_{F_{\alpha(m)}}+\left\|y_{p}\right\|_{F_{p}}\right] .
\end{aligned}
$$

So, $\left(z_{N}\right)$ is a Cauchy sequence in $E_{n}$ for any $n \geq 0$. Since $E_{n}$ is a Banach space, $\left(z_{N}\right)$ tends to $z$ in $E_{n}$ for any $n$ and more precisely with $z \in B_{E_{n}}\left(a_{m}, 2 r_{m}\right)$ for any $n \geq 0$. We get:

$$
z_{N}=z_{N-1}-\left[D f\left(a_{m}\right)\right]^{-1}\left(f\left(z_{N-1}\right)-f(0)-y_{p}\right) .
$$

So:

$$
z=z-\left[D f\left(a_{m}\right)\right]^{-1}\left(f(z)-f(0)-y_{p}\right)
$$

Therefore:

$$
f(z)=y_{p}+f(0) .
$$

Now, we consider any $Y$ in $B_{F_{n}}\left(f\left(a_{m}\right)-f(0), \frac{r_{m}^{\prime}}{\alpha_{m}\left\|a_{m}\right\|_{E_{m}}^{r}+\beta_{m}}\right)$ for a certain $n \geq 0$. With assumptions $9 \mathrm{c})$, we get a sequence $\left(Y_{p}\right)$ with:

$$
Y_{p} \in B_{F_{n}}\left(f\left(a_{m}\right)-f(0), \frac{r_{m}^{\prime}}{\alpha_{m}\left\|a_{m}\right\|_{E_{m}}^{r}+\beta_{m}}\right) \cap C_{p}
$$

such that $Y_{p} \rightarrow Y$ for the $F_{n}$ norm. Then, for each $Y_{p}$, there exists $Z_{p}$ in $B_{E_{n}}\left(a_{m}, 2 r_{m}^{\prime}\right)$ (here, for any $n \geq 0$ ), such that:

$$
f\left(Z_{p}\right)=Y_{p}+f(0) .
$$

According to assumption 7), now, we get:

$$
\left\|Z_{p}-Z_{q}\right\|_{E_{n+1}} \leq c_{n}\left(Z_{p}, Z_{q}\right)\left\|Y_{p}-Y_{q}\right\|_{F_{n}} .
$$

Since $c_{n}$ is continuous from $E_{n+1} \times E_{n+1}$ into $\mathbb{R}^{+}$and since $Z_{p}$ is bounded in $E_{n+1}$, we obtain:

$$
\left\|Z_{p}-Z_{q}\right\|_{E_{n+1}} \leq C\left\|Y_{p}-Y_{q}\right\|_{F_{n}}
$$

where $C$ denotes a certain constant. Since $\left(Y_{p}\right)$ is a Cauchy sequence in $F_{n},\left(Z_{p}\right)$ is a Cauchy sequence in $E_{n+1}$ so that $\left(Z_{p}\right)$ tends to a $Z$ in $\overline{B_{E_{n+1}}\left(a_{m}, 2 r_{m}^{\prime}\right)}$ that is in $B_{E_{n+1}}\left(a_{m}, 2 r_{m}\right)$. So, $f\left(Z_{p}\right)$ tends to $f(Z)$ in $\mathcal{F}_{n-1}$. Since $f\left(Z_{p}\right)$ tends to $Y+f(0)$ in $F_{n}$, by the fact that $F_{n} \subset \mathcal{F}_{n} \subset \mathcal{F}_{n-1}$ with continuous injections, we get that $f(Z)=Y+f(0)$. Moreover, the uniqueness of $Z$ is guaranteed by assumption 7 .

Therefore, for any $a_{m}$ in $A_{m}$ (this for any $m \geq 0$ ) and for any $n \geq 0, f^{-1}$ exists from $B_{F_{n}}\left(f\left(a_{m}\right), \frac{r_{m}}{\alpha_{m}\left\|a_{m}\right\|_{E_{m}}^{r}+\beta_{m}}\right)$ into $B_{E_{n+1}}\left(a_{m}, 2 r_{m}\right)$, for any $r_{m}$ such that:

$$
r_{m}<\frac{1}{4\left(\alpha_{m}\left\|a_{m}\right\|_{E_{m}}^{r}+\beta_{m}\right) \times \gamma\left(2\left\|a_{m}\right\|_{E_{m}}+3\right)} .
$$

\section{Application to the Yang-Mills equation}

3.1. Lorentz metric. We are going to show the existence (but not the uniqueness) of smooth Landau-Lorentz gauge fields solutions to the Yang-Mills equation in a smooth compact manifold with boundary $P$ in $\mathbb{R}^{3}$ for a finite interval of time $[0, T], T>0$ or in the whole $\mathbb{R}^{4}$. We consider:

$$
\rho_{P}=\max _{x \in P}|x| .
$$


We conceive a torus $\Omega$ equal to $\left.\mathbb{R}^{3}\right|_{((2 N+1) \mathbb{Z})^{3}}$, with an integer $N$ such that:

$$
\rho_{P}<N \text {. }
$$

On $] 0, T[\times \Omega$, we will work with the simplest Lorentz metric:

$$
\left(\begin{array}{ll}
-1 & 0 \\
0 & I d_{3}
\end{array}\right)
$$

where $I d_{3}$ denotes the identity matrix in dimension three. So, in local chart $x=$ $\left(x_{0}, x_{1}, x_{2}, x_{3}\right), x_{0}$ will denote the time (" $t$ " and " $s$ " too) and $\left(x_{1}, x_{2}, x_{3}\right)$ the space coordinates. In this metrics, we get:

$$
\partial^{\mu} \partial_{\mu} V=-\partial_{0}^{2} V+\partial_{1}^{2} V+\partial_{2}^{2} V+\partial_{3}^{2} V
$$

that is, the opposite of the Dalembertian operator.

A couple of cases can be considered: the case of the classical Yukawa-KleinYang-Mills theory with commutators and the case of some super-symmetric YangMills fields. Even if the second case is a generalization of the first one, we prefer to begin with the easiest one for a couple of reasons: the calculations of the first case will be largely used in the second one and the first one, which is the historical one, involves the electromagnetic, the weak and the strong forces.

\subsection{Classical strength tensors with commutators.}

3.2.1. Introduction of the Yang-Mills equation of the motion. In $] 0, T[\times \Omega$, we consider the gauge field $x \rightarrow\left(\chi_{a}^{\mu}+A_{a}^{\mu}\right)(x)$, where $\chi_{a}^{\mu}$ denotes complex constants, that is $\left(\chi^{\mu}+A^{\mu}\right)(x)=\left(\chi_{a}^{\mu}(x)+A_{a}^{\mu}(x)\right) t^{a}$, with $0 \leq \mu \leq 3,1 \leq a \leq a^{\prime}$ in the summation, where $a^{\prime}$ is the number of generators $t_{a}$ of the Lie algebra associated with the gauge field $\chi+A$. The use of a constant field $\chi$ is necessary to prove the existence of an infinity of Landau-Lorentz Yang-Mills fields. In this paragraph 3, we consider the strength tensors $F_{\mu \nu}$ defined by a Lie bracket:

$$
F_{\mu \nu}=\partial_{\mu}\left(\chi_{\nu}+A_{\nu}\right)-\partial_{\nu}\left(\chi_{\mu}+A_{\mu}\right)-g\left[\chi_{\mu}+A_{\mu}, \chi_{\nu}+A_{\nu}\right], \quad 0 \leq \mu, \nu \leq 3,
$$

where $g$ denotes the Yukawa coupling constant (for us, any complex number). We suppose the classical relations of commutation inside the Lie algebra stated with the classical Christoffel complex numbers $C_{c}^{a b}$ :

$$
\left[t^{a}, t^{b}\right]=C_{c}^{a b} t^{c} .
$$

The Christoffel numbers are antisymmetric:

$$
C_{c}^{a b}=-C_{a b}^{c} .
$$

We consider the covariant derivative $D_{\mu}$ expressed by:

$$
D_{\mu}=\partial_{\mu} \cdot-g\left[\chi_{\mu}+A_{\mu}, .\right] .
$$

Then, the non homogeneous Yang-Mills equation of motion of the gauge field is:

$$
D^{\mu} F_{\mu \nu}=\partial^{\mu} F_{\mu \nu}-g\left[\chi^{\mu}+A^{\mu}, F_{\mu \nu}\right]=0, \quad \nu=0,1,2,3,
$$

where $g$ still denotes the Yukawa coupling constant. We consider the following equation, where $A$ is naturally the unknown field:

$$
\begin{aligned}
& {\left[\partial^{\mu} \partial_{\mu} A_{\nu a}-g C_{a}^{b c}\left(\partial^{\mu} A_{\mu b}\right)\left(\chi_{\nu c}+A_{\nu c}\right)-2 g C_{a}^{b c}\left(\chi_{\mu b}+A_{\mu b}\right) \partial^{\mu} A_{\nu c}\right.} \\
& +g C_{a}^{b c}\left(\chi^{\mu b}+A^{\mu b}\right) \partial_{\nu} A_{\mu c}+g^{2} C_{a}^{d e}\left(\chi^{\mu d}+A^{\mu d}\right)\left(C_{e}^{b c}\left(\chi_{\mu b}+A_{\mu b}\right)\right. \\
& \left.\left.\times\left(\chi_{\nu c}+A_{\nu c}\right)\right)\right] t^{a}=0, \quad 1 \leq \nu \leq 3, \quad 1 \leq a \leq a^{\prime} .
\end{aligned}
$$


The link between (2) and (3) is the following one: if we denote by $\left(Y M\left(A_{\nu a}\right)\right) t^{a}$ the left-member of $(2)$, then (3) is:

$$
\left(Y M\left(A_{\nu a}\right)+\partial_{\nu} \partial^{\mu} A_{\mu a}\right) t^{a}=0 .
$$

Then, if we are able to find a field $A$ which satisfies (4), it will only remain to show that $\chi+A$ is a Landau-Lorentz field, that is $\partial^{\mu}\left(\chi_{\mu a}+A_{\mu a}\right)=0$, for any $a$ with $1 \leq a \leq a^{\prime}$, to obtain a solution $A$ to the Yang-Mills equation (2).

Now, we recall that the generators $t_{a}$ of the Lie algebra are such that:

$$
\left(\lambda^{a} t_{a}=0\right) \Longleftrightarrow\left(\lambda_{a}=0, \quad \text { for every } a\right) .
$$

So, if we denote by $\left(Y M_{w}\left(A_{\nu a}\right)\right) t^{a}$ the left member of (4), we just have (for the moment) to find a solution $A$ to:

$$
Y M_{w}\left(A_{\nu a}\right)=0, \quad \text { for } 0 \leq \nu \leq 3, \quad 1 \leq a \leq a^{\prime} .
$$

3.2.2. Introduction of the Yang-Mills derivative operator. The formal differential operator $d Y M_{w} \mathcal{A}$ of $Y M_{w}$, taken in some gauge field $\mathcal{A}$ whose regularity will be later explained, is:

$$
\begin{aligned}
& \text { (7) } d Y M_{w \mathcal{A}} A_{\nu a}=\partial^{\mu} \partial_{\mu} A_{\nu a}-g C_{a}^{b c}\left(\partial^{\mu} \mathcal{A}_{\mu b}\right) A_{\nu c}-g C_{a}^{b c} \mathcal{A}_{\nu c} \partial^{\mu} A_{\mu b} \\
& -2 g C_{a}^{b c}\left(\partial^{\mu} \mathcal{A}_{\nu c}\right) A_{\mu b}-2 g C_{a}^{b c} \mathcal{A}_{\mu b} \partial^{\mu} A_{\nu c}+g C_{a}^{b c} \mathcal{A}^{\mu b} \partial_{\nu} A_{\mu c}+g C_{a}^{b c}\left(\partial_{\nu} \mathcal{A}_{\mu c}\right) A^{\mu b} \\
& +g^{2} C_{a}^{d e} \mathcal{A}^{\mu d}\left(C_{e}^{b c} \mathcal{A}_{\mu b} A_{\nu c}\right)+g^{2} C_{a}^{d e} \mathcal{A}^{\mu d}\left(C_{e}^{b c} \mathcal{A}_{\nu c} A_{\mu b}\right)+g^{2} C_{a}^{d e}\left(C_{e}^{b c} \mathcal{A}_{\mu b} \mathcal{A}_{\nu c}\right) A^{\mu d} \\
& -g C_{a}^{b c} \chi_{\nu c} \partial^{\mu} A_{\mu b}-2 g C_{a}^{b c} \chi_{\mu b} \partial^{\mu} A_{\nu c}+g C_{a}^{b c} \chi^{\mu b} \partial_{\nu} A_{\mu c}+g^{2} C_{a}^{d e} \chi^{\mu d}\left(C_{e}^{b c} \mathcal{A}_{\mu b} A_{\nu c}\right) \\
& +g^{2} C_{a}^{d e} \mathcal{A}^{\mu d}\left(C_{e}^{b c} \chi_{\mu b} A_{\nu c}\right)+g^{2} C_{a}^{d e} \chi^{\mu d}\left(C_{e}^{b c} \chi_{\mu b} A_{\nu c}\right)+g^{2} C_{a}^{d e} \chi^{\mu d}\left(C_{e}^{b c} \mathcal{A}_{\nu c} A_{\mu b}\right) \\
& +g^{2} C_{a}^{d e} \mathcal{A}^{\mu d}\left(C_{e}^{b c} \chi_{\nu c} A_{\mu b}\right)+g^{2} C_{a}^{d e} \chi^{\mu d}\left(C_{e}^{b c} \chi_{\nu c} A_{\mu b}\right)+g^{2} C_{a}^{d e}\left(C_{a}^{b c} \chi_{\mu b} \mathcal{A}_{\nu c}\right) A^{\mu d} \\
& +g^{2} C_{a}^{d e}\left(C_{e}^{b c} \mathcal{A}_{\mu b} \chi_{\nu c}\right) A^{\mu d}+g^{2} C_{a}^{d e}\left(C_{e}^{b c} \chi_{\mu b} \chi_{\nu c}\right) A^{\mu d}
\end{aligned}
$$

for $0 \leq \nu \leq 3,1 \leq a \leq a^{\prime}$. So, $Y M, Y M_{w}$ and $d Y M_{w, \mathcal{A}}$ depend on the constant field $\chi=\left(\chi_{\nu a}\right)_{0 \leq \nu \leq 3,1 \leq a \leq a^{\prime}}$. We will denote:

$$
\left\{\begin{array}{l}
Y M_{w} A=\left(Y M_{w} A_{01}, Y M_{w} A_{11}, \ldots, Y M_{w} A_{2 a^{\prime}}, Y M_{w} A_{3 a^{\prime}}\right) \\
d Y M_{w \mathcal{A}} A=\left(d Y M_{w \mathcal{A}} A_{01}, d Y M_{w \mathcal{A}} A_{11}, \ldots, d Y M_{w \mathcal{A}} A_{2 a^{\prime}}, d Y M_{w \mathcal{A}} A_{3 a^{\prime}}\right)
\end{array} .\right.
$$

3.2.3. Assumptions 1, 3 and 9. We consider the spaces:

$$
\left\{\begin{aligned}
{ }^{4 a^{\prime}} H^{k}(] 0, T[\times \Omega)= & \left\{u=\left(u_{1}, u_{2}, \ldots, u_{4 a^{\prime}}\right), u_{i} \in H^{k}(] 0, T[\times \Omega),\right. \\
& \left.1 \leq i \leq 4 a^{\prime}\right\}, \quad \text { for } k \geq 0 \\
{ }^{4 a^{\prime}} H_{m}^{k}(] 0, T[\times \Omega)= & \left\{u \in{ }^{4 a^{\prime}} H^{k}(] 0, T[\times \Omega), \partial_{0}^{i}\left(0, x_{1}, x_{2}, x_{3}\right)=0, i=0,1,\right. \\
& \ldots, m\}, \quad 0 \leq m \leq k-3
\end{aligned}\right.
$$

Relatively to the statement of Theorem 2.1, we put:

$$
\left\{\begin{array}{ll}
\mathcal{F}_{n}={ }^{4 a^{\prime}} H^{n+6}(] 0, T[\times \Omega) & (n \geq 0) \\
E_{n}={ }^{4 a^{\prime}} H_{3}^{n+6}(] 0, T[\times \Omega) & (n \geq 0) \\
F_{n}={ }^{4 a^{\prime}} H_{1}^{n+6}(] 0, T[\times \Omega) & (n \geq 0)
\end{array} .\right.
$$

We consider the sets $\left(A_{n}\right)_{n \geq 0}$ of polynomials $P$ of the variables $x_{0}, x_{1}, x_{2}$ and $x_{3}$ of degrees $\leq n$, with:

$$
\partial_{0}^{i} P\left(0, x_{1}, x_{2}, x_{3}\right)=0, \quad i=0,1,2,3 .
$$


We consider the sets $\left(C_{n}\right)_{n \geq 0}$ of polynomials $P$ of the variables $x_{0}, x_{1}, x_{2}$ and $x_{3}$ of degrees $\leq n$, with:

$$
\partial_{0}^{i} P\left(0, x_{1}, x_{2}, x_{3}\right)=0, \quad i=0,1 .
$$

Now, let's check if all the assumptions of Theorem 1 are fulfilled. So, assumptions 1,3 and 9 are evidently fulfilled.

3.2.4. Assumptions 2 and 5. Relatively to Theorem 2.1, we consider $f=Y M_{w}$.

Does $Y M_{w}$ act continuously from ${ }^{4 a^{\prime}} H_{3}^{n+2}(] 0, T[\times \Omega)$ into ${ }^{4 a^{\prime}} H^{n}(] 0, T[\times \Omega)$ for $n \geq 6$ ? Consider a couple of gauge fields $A$ and $C$ in ${ }^{4 a^{\prime}} H_{3}^{n+2}(] 0, T[\times \Omega)$ (we have to notice that $C$ has not to be confused with the Christoffel coefficients $C_{a}^{b c}$ ). We get:

$$
\begin{aligned}
& Y M_{w} A_{\nu a}-Y M_{w} C_{\nu a}=\partial^{\mu} \partial_{\mu}\left(A_{\nu a}-C_{\nu a}\right)-g C_{a}^{b c}\left(\partial^{\mu} A_{\mu b}-\partial^{\mu} C_{\mu b}\right)\left(\chi_{\nu c}+A_{\nu c}\right) \\
& +g C_{a}^{b c} \partial^{\mu} C_{\mu b}\left(C_{\nu c}-A_{\nu c}\right)-2 g C_{a}^{b c}\left(\chi_{\mu b}+A_{\mu b}\right)\left(\partial^{\mu} A_{\nu c}-\partial^{\mu} C_{\nu c}\right) \\
& +2 g C_{a}^{b c}\left(C_{\mu b}-A_{\mu b}\right) \partial^{\mu} C_{\nu c}+g C_{a}^{b c}\left(\chi^{\mu b}+A^{\mu b}\right)\left(\partial_{\nu} A_{\mu c}-\partial_{\nu} C_{\mu c}\right) \\
& -g C_{a}^{b c}\left(C^{\mu b}-A^{\mu b}\right) \partial_{\nu} C_{\mu c}+g^{2} C_{a}^{d e}\left(A^{\mu d}-C^{\mu d}\right) C_{e}^{b c}\left(\chi_{\mu b}+A_{\mu b}\right)\left(\chi_{\nu c}+A_{\nu c}\right) \\
& -g^{2} C_{a}^{d e}\left(\chi^{\mu d}+C^{\mu d}\right) C_{e}^{b c}\left[\left(C_{\mu b}-A_{\mu b}\right)\left(\chi_{\nu c}+C_{\nu c}\right)-\left(\chi_{\mu b}+A_{\mu b}\right)\left(A_{\nu c}-C_{\nu c}\right)\right] .
\end{aligned}
$$

In the following paragraphs, we use the Cauchy-Schwarz-Buniakowski inequality and Sobolev imbeddings with continuous injections:

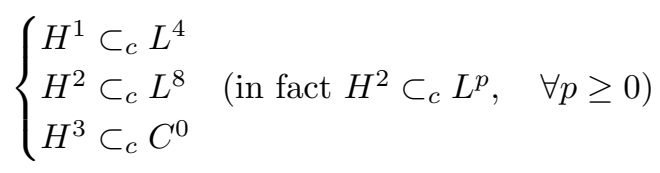

valuable for the four-dimensional $\mathbb{R} \times \Omega$. Now, we evidently get:

$$
\begin{aligned}
& \left\|\partial^{\mu} \partial_{\mu}\left(A_{\nu a}-C_{\nu a}\right)\right\|_{H^{n}(] 0, T[\times \Omega)} \leq\left\|A_{\nu a}-C_{\nu a}\right\|_{H^{n+2}(] 0, T[\times \Omega)} \\
& \left\|\partial^{\mu} \partial_{\mu}\left(A_{\nu a}-C_{\nu a}\right)\right\|_{H^{n}(] 0, T[\times \Omega)} \leq\|A-C\|_{4 a^{\prime} H^{n+2}(] 0, T[\times \Omega)} .
\end{aligned}
$$

In the following calculations, the constants $K$ or else can change from a line to another.

We get too:

$$
\begin{aligned}
& \left\|\left(\chi_{\mu b}+A_{\mu b}\right)\left(\partial_{\mu} A_{\nu c}-\partial_{\mu} C_{\nu c}\right)\right\|_{H^{n}(] 0, T[\times \Omega)} \leq \\
& K \sum_{|\alpha| \leq n} \sum_{\substack{\gamma+\delta=\alpha \\
|\delta|<n}}\left\|\partial_{\gamma}\left(\chi_{\mu b}+A_{\mu b}\right) \partial_{\delta} \partial_{\mu}\left(A_{\nu c}-C_{\nu c}\right)\right\|_{L^{2}(] 0, T[\times \Omega)} \\
& +K \sum_{|\delta|=n}\left\|\left(\chi_{\mu b}+A_{\mu b}\right) \partial_{\delta} \partial_{\mu}\left(A_{\nu c}-C_{\nu c}\right)\right\|_{L^{2}(] 0, T[\times \Omega)} .
\end{aligned}
$$

For $|\delta|<n$ :

$$
\begin{aligned}
& \left\|\partial_{\gamma}\left(\chi_{\mu b}+A_{\mu b}\right) \partial_{\delta} \partial_{\mu}\left(A_{\nu c}-C_{\nu c}\right)\right\|_{L^{2}(] 0, T[\times \Omega)} \\
& \quad \leq\left\|\partial_{\gamma}\left(\chi_{\mu b}+A_{\mu b}\right)\right\|_{L^{4}(] 0, T[\times \Omega)}\left\|\partial_{\delta} \partial_{\mu}\left(A_{\nu c}-C_{\nu c}\right)\right\|_{L^{4}(] 0, T[\times \Omega)} \\
& \left\|\partial_{\gamma}\left(\chi_{\mu b}+A_{\mu b} \partial_{\delta} \partial_{\mu}\left(A_{\nu c}\right)-C_{\nu c}\right)\right\|_{L^{2}(] 0, T[\times \Omega)} \\
& \quad \leq K\left\|\chi_{\mu b}+A_{\mu b}\right\|_{H^{n+2}(] 0, T[\times \Omega)}\left\|A_{\nu c}-C_{\nu c}\right\|_{H^{n+2}(] 0, T[\times \Omega)} .
\end{aligned}
$$


For $|\delta|=n$ :

$$
\begin{aligned}
\left\|\left(\chi_{\mu b}+A_{\mu b}\right) \partial_{\delta} \partial_{\mu}\left(A_{\nu c}-C_{\nu c}\right)\right\|_{L^{2}(] 0, T[\times \Omega)} \leq & \left\|\chi_{\mu b}+A_{\mu b}\right\|_{H^{3}(] 0, T[\times \Omega)} \\
& \times\left\|A_{\nu c}-C_{\nu c}\right\|_{H^{n+2}(] 0, T[\times \Omega)} .
\end{aligned}
$$

Since $n \geq 6, n+2 \geq 3$, so that:

$$
\begin{aligned}
& \left\|-2 g C_{a}^{b c}\left(\chi_{\mu b}+A_{\mu b}\right)\left(\partial^{\mu} A_{\nu c}-\partial^{\mu} C_{\nu c}\right)\right\|_{H^{n}(] 0, T[\times \Omega)} \\
& \leq K\|\chi+A\|_{4 a^{\prime} H^{n+2}(] 0, T[\times \Omega)}\|A-C\|_{4 a^{\prime} H^{n+2}(] 0, T[\times \Omega)} .
\end{aligned}
$$

By similar treatments:

$$
\begin{aligned}
& \|-g C_{a}^{b c}\left(\partial^{\mu} A_{\mu b}-\partial^{\mu} C_{\mu b}\right)\left(\chi_{\nu c}+A_{\nu c}\right) \\
& +g C_{a}^{b c}\left(\chi^{\mu b}+A^{\mu b}\right)\left(\partial_{\nu} A_{\mu c}-\partial_{\nu} C_{\mu c}\right) \|_{H^{n}(] 0, T[\times \Omega)} \\
& \leq K\|\chi+A\|_{4 a^{\prime} H^{n+2}(] 0, T[\times \Omega)} \times\|A-C\|_{4 a^{\prime} H^{n+2}(] 0, T[\times \Omega)} .
\end{aligned}
$$

Moreover:

$$
\begin{aligned}
& \left\|2 g C_{a}^{b c}\left(C_{\mu b}-A_{\mu b}\right) \partial^{\mu} C_{\nu c}\right\|_{H^{n}(] 0, T[\times \Omega)} \\
& \leq K\|C-A\|_{4 a^{\prime} H^{n+2}(] 0, T[\times \Omega)}\|C\|_{4 a^{\prime} H^{n+2}(] 0, T[\times \Omega)} .
\end{aligned}
$$

We get too:

$$
\begin{aligned}
& \| g C_{a}^{b c} \partial^{\mu} C_{\mu b}\left(C_{\nu c}-A_{\nu c}\right) \\
& -g C_{a}^{b c}\left(C^{\mu b}-A^{\mu b}\right) \partial_{\nu} C_{\mu c} \|_{H^{n}(] 0, T[\times \Omega)} \\
& \leq K\|C\|_{4 a^{\prime} H^{n+2}(] 0, T[\times \Omega)} \times\|A-C\|_{4 a^{\prime} H^{n+2}(] 0, T[\times \Omega)} .
\end{aligned}
$$

Now, let's study the case of $\left(A_{\mu d}-C_{\mu d}\right)\left(\chi_{\mu b}+A_{\mu b}\right)\left(\chi_{\nu c}+A_{\nu c}\right)$ :

$$
\begin{aligned}
& \left\|\left(A_{\mu d}-C_{\mu d}\right)\left(\chi_{\mu b}+A_{\mu b}\right)\left(\chi_{\nu c}+A_{\nu c}\right)\right\|_{H^{n}(] 0, T[\times \Omega)} \leq \\
& C \sum_{|\alpha| \leq n \gamma+\delta=\alpha} \sum_{\kappa} \| \partial_{\gamma}\left(A_{\mu d}-C_{\mu d}\right) \\
& \quad \sum_{\kappa+\tau=\delta} \partial_{\kappa}\left(\chi_{\mu b}+A_{\mu b}\right) \partial_{\tau}\left(\chi_{\nu c}+A_{\nu c}\right) \|_{L^{2}(] 0, T[\times \Omega)}
\end{aligned}
$$

and so:

$$
\begin{aligned}
& \left\|\partial_{\gamma}\left(A_{\mu d}-C_{\mu d}\right) \times \partial_{\kappa}\left(\chi_{\mu b}+A_{\mu b}\right) \times \partial_{\tau}\left(\chi_{\nu c}+A_{\nu c}\right)\right\|_{L^{2}(] 0, T[\times \Omega)} \\
& \leq\left\|\partial_{\gamma}\left(A_{\mu d}-C_{\mu d}\right)\right\|_{L^{8}(] 0, T[\times \Omega)}\left\|\partial_{\kappa}(\chi+A)\right\|_{4 a^{\prime} L^{8}(] 0, T[\times \Omega)} \\
& \times\left\|\partial_{\tau}(\chi+A)\right\|_{4 a^{\prime} L^{4}(] 0, T[\times \Omega)} \\
& \left\|\partial_{\gamma}\left(A_{\mu d}-C_{\mu d}\right) \times \partial_{\kappa}\left(\chi_{\mu b}+A_{\mu b}\right) \times \partial_{\tau}\left(\chi_{\nu c}+A_{\nu c}\right)\right\|_{L^{2}(] 0, T[\times \Omega)} \\
& \leq K\|A-C\|_{4 a^{\prime} H^{n+2}(] 0, T[\times \Omega)}\|\chi+A\|_{4 a^{\prime} H^{n+2}(] 0, T[\times \Omega)} \\
& \|\chi+A\|_{4 a^{\prime} H^{n+2}(] 0, T[\times \Omega)} .
\end{aligned}
$$


So:

$$
\begin{aligned}
& \| g^{2} C_{a}^{d e}\left(A^{\mu d}-C^{\mu d}\right) C_{e}^{b c}\left(\chi_{\mu b}+A_{\mu b}\right)\left(\chi_{\nu c}+A_{\nu c}\right)-g^{2} C_{a}^{d e}\left(\chi^{\mu d}+C^{\mu d}\right) C_{e}^{b c} \\
& \times\left[\left(C_{\mu b}-A_{\mu b}\right)\left(\chi_{\nu c}+C_{\nu c}\right)-\left(\chi_{\mu b}+A_{\mu b}\right)\left(A_{\nu c}-C_{\nu c}\right)\right] \|_{H^{n}(] 0, T[\times \Omega)} \\
& \leq K\|A-C\|_{4 a^{\prime} H^{n+2}(] 0, T[\times \Omega)}\left(\|\chi+A\|_{4 a^{\prime} H^{n+2}(] 0, T[\times \Omega)}^{2}\right. \\
& \left.+\|\chi+C\|_{4 a^{\prime} H^{n+2}(] 0, T[\times \Omega)}^{2}\right) .
\end{aligned}
$$

So, $f$ acts continuously from $E_{n+2}$ into $\mathcal{F}_{n}$ for $n \geq 0$. Moreover, if:

$$
\begin{cases}\partial_{0}^{i} A_{k a}\left(0, x_{1}, x_{2}, x_{3}\right)=0, & 0 \leq i, k \leq 3, \quad 1 \leq a \leq a^{\prime} \\ \partial_{0}^{i} C_{k a}\left(0, x_{1}, x_{2}, x_{3}\right)=0, & 0 \leq i, k \leq 3, \quad 1 \leq a \leq a^{\prime}\end{cases}
$$

then:

$$
\left\{\begin{array}{l}
\partial_{0}^{i}\left(\partial_{j}^{2} A_{k a}\right)\left(0, x_{1}, x_{2}, x_{3}\right)=\partial_{j}^{2} \partial_{0}^{i} A_{k a}\left(0, x_{1}, x_{2}, x_{3}\right)=0, \quad i=0,1 \\
\partial_{0}^{i}\left(\partial_{j}^{2} C_{k a}\right)\left(0, x_{1}, x_{2}, x_{3}\right)=0, \quad i=0,1
\end{array}\right.
$$

and:

$$
\left\{\begin{array}{l}
\left(\partial_{j} A_{k a} \times A_{l b}\right)\left(0, x_{1}, x_{2}, x_{3}\right)=\left(\partial_{j} A_{k a}\left(0, x_{1}, x_{2}, x_{3}\right)\right) \times A_{l b}\left(0, x_{1}, x_{2}, x_{3}\right)=0 \\
\partial_{0}\left(\partial_{j} A_{k a} \times A_{l b}\right)\left(0, x_{1}, x_{2}, x_{3}\right)=\left(\partial_{j} \partial_{0} A_{k a}\left(0, x_{1}, x_{2}, x_{3}\right)\right) \times A_{l b}\left(0, x_{1}, x_{2}, x_{3}\right) \\
+\left(\partial_{j} A_{k a}\right)\left(0, x_{1}, x_{2}, x_{3}\right) \times\left(\partial_{0} A_{l b}\right)\left(0, x_{1}, x_{2}, x_{3}\right)=0
\end{array}\right.
$$$$
\left\{\begin{array}{l}
\left(\partial_{j} C_{k a} \times C_{l b}\right)\left(0, x_{1}, x_{2}, x_{3}\right)=\left(\partial_{j} C_{k a}\left(0, x_{1}, x_{2}, x_{3}\right)\right) \times C_{l b}\left(0, x_{1}, x_{2}, x_{3}\right)=0 \\
\partial_{0}\left(\partial_{j} C_{k a} \times C_{l b}\right)\left(0, x_{1}, x_{2}, x_{3}\right)=\left(\partial_{j} \partial_{0} C_{k a}\left(0, x_{1}, x_{2}, x_{3}\right)\right) \times C_{l b}\left(0, x_{1}, x_{2}, x_{3}\right) \\
+\left(\partial_{j} C_{k a}\right)\left(0, x_{1}, x_{2}, x_{3}\right) \times\left(\partial_{0} C_{l b}\right)\left(0, x_{1}, x_{2}, x_{3}\right)=0
\end{array}\right.
$$$$
\left\{\begin{array}{l}
\left(\partial_{j} A_{k a} \times \chi_{l b}\right)\left(0, x_{1}, x_{2}, x_{3}\right)=\left(\partial_{j} A_{k a}\left(0, x_{1}, x_{2}, x_{3}\right)\right) \times \chi_{l b}\left(0, x_{1}, x_{2}, x_{3}\right)=0 \\
\partial_{0}\left(\partial_{j} A_{k a} \times \chi_{l b}\right)\left(0, x_{1}, x_{2}, x_{3}\right)=\left(\partial_{j} \partial_{0} A_{k a}\left(0, x_{1}, x_{2}, x_{3}\right)\right) \times \chi_{l b}\left(0, x_{1}, x_{2}, x_{3}\right) \\
+\left(\partial_{j} A_{k a}\right)\left(0, x_{1}, x_{2}, x_{3}\right) \times\left(\partial_{0} \chi_{l b}\right)\left(0, x_{1}, x_{2}, x_{3}\right)=0
\end{array}\right.
$$$$
\left\{\begin{array}{l}
\left(\partial_{j} C_{k a} \times A_{l b}\right)\left(0, x_{1}, x_{2}, x_{3}\right)=\left(\partial_{j} C_{k a}\left(0, x_{1}, x_{2}, x_{3}\right)\right) \times A_{l b}\left(0, x_{1}, x_{2}, x_{3}\right)=0 \\
\partial_{0}\left(\partial_{j} C_{k a} \times A_{l b}\right)\left(0, x_{1}, x_{2}, x_{3}\right)=\left(\partial_{j} \partial_{0} C_{k a}\left(0, x_{1}, x_{2}, x_{3}\right)\right) \times A_{l b}\left(0, x_{1}, x_{2}, x_{3}\right) \\
+\left(\partial_{j} C_{k a}\right)\left(0, x_{1}, x_{2}, x_{3}\right) \times\left(\partial_{0} A_{l b}\right)\left(0, x_{1}, x_{2}, x_{3}\right)=0
\end{array}\right.
$$$$
\left\{\begin{array}{l}
\left(\partial_{j} C_{k a} \times \chi_{l b}\right)\left(0, x_{1}, x_{2}, x_{3}\right)=\left(\partial_{j} C_{k a}\left(0, x_{1}, x_{2}, x_{3}\right)\right) \times \chi_{l b}\left(0, x_{1}, x_{2}, x_{3}\right)=0 \\
\partial_{0}\left(\partial_{j} C_{k a} \times \chi_{l b}\right)\left(0, x_{1}, x_{2}, x_{3}\right)=\left(\partial_{j} \partial_{0} C_{k a}\left(0, x_{1}, x_{2}, x_{3}\right)\right) \times \chi_{l b}\left(0, x_{1}, x_{2}, x_{3}\right) \\
+\left(\partial_{j} C_{k a}\right)\left(0, x_{1}, x_{2}, x_{3}\right) \times\left(\partial_{0} \chi_{l b}\right)\left(0, x_{1}, x_{2}, x_{3}\right)=0
\end{array}\right.
$$ 
and:

$$
\left\{\begin{array}{l}
\left(A_{j a}-C_{j a}\right)\left(\chi_{k b}+A_{k b}\right)\left(\chi_{l c}+A_{l c}\right)\left(0, x_{1}, x_{2}, x_{3}\right) \\
=\left(A_{j a}-C_{j a}\right)\left(0, x_{1}, x_{2}, x_{3}\right) \\
\times\left(\chi_{k b}+A_{k b}\right)\left(0, x_{1}, x_{2}, x_{3}\right) \times\left(\chi_{l c}+A_{l c}\right)\left(0, x_{1}, x_{2}, x_{3}\right)=0 \\
\partial_{0}\left(\left(A_{j a}-C_{j a}\right) \times\left(\chi_{k b}+A_{k b}\right) \times\left(\chi_{l c}+A_{l c}\right)\right)\left(0, x_{1}, x_{2}, x_{3}\right) \\
=\left(\partial_{0} A_{j a}-\partial_{0} C_{j a}\right)\left(0, x_{1}, x_{2}, x_{3}\right) \\
\times\left(\chi_{k b}+A_{k b}\right)\left(0, x_{1}, x_{2}, x_{3}\right) \times\left(\chi_{l c}+A_{l c}\right)\left(0, x_{1}, x_{2}, x_{3}\right) \\
+\left(A_{j a}-C_{j a}\right)\left(0, x_{1}, x_{2}, x_{3}\right) \times\left(\partial_{0}\left(\chi_{k b}+A_{k b}\right)\left(0, x_{1}, x_{2}, x_{3}\right)\right) \\
\times\left(\chi_{l c}+A_{l c}\right)\left(0, x_{1}, x_{2}, x_{3}\right) \\
+\left(A_{j a}-C_{j a}\right)\left(0, x_{1}, x_{2}, x_{3}\right) \times\left(\chi_{k b}+A_{k b}\right)\left(0, x_{1}, x_{2}, x_{3}\right) \\
\times\left(\partial_{0}\left(\chi_{l c}+A_{l c}\right)\left(0, x_{1}, x_{2}, x_{3}\right)\right)=0 .
\end{array}\right.
$$

So, $f(A)-f(C)$ acts from $E_{n+2}^{2}$ into $F_{n}$ for $n \geq 0$. Now, for any $\mathcal{A}$ in $E_{n+2}$, is $d Y M_{w \mathcal{A}}$ in $L\left(E_{n+2}, F_{n}\right)$ ? We get:

$$
\left\|\partial^{\mu} \partial_{\mu} A_{\nu a}\right\|_{H^{n}(] 0, T[\times \Omega)} \leq\|A\|_{4 a^{\prime} H^{n+2}(] 0, T[\times \Omega)} .
$$

We get too, by using the calculations done on $Y M_{w} \mathcal{A}_{\nu a}-Y M_{w} C_{\nu a}$ :

$$
\begin{aligned}
& \left\|-g C_{a}^{b c}\left(\partial^{\mu} \mathcal{A}_{\mu b}\right) A_{\nu c}\right\|_{H^{n}(] 0, T[\times \Omega)} \\
& \leq K\|\mathcal{A}\|_{4 a^{\prime} H^{n+2}(] 0, T[\times \Omega)} \times\|A\|_{4 a^{\prime} H^{n+2}(] 0, T[\times \Omega)}
\end{aligned}
$$

and idem for the terms from $-g C_{a}^{b c} \mathcal{A}_{\nu c} \partial^{\mu} A_{\mu b}$ to $g C_{a}^{b c}\left(\partial_{\nu} \mathcal{A}_{\mu c}\right) A^{\mu b}$ in (7). Now, following the calculation done above, we obtain:

$$
\begin{aligned}
& \left\|g^{2} C_{a}^{d e} \mathcal{A}^{\mu d}\left(C_{e}^{b c} \mathcal{A}_{\mu b} A_{\nu c}\right)\right\|_{H^{n}(] 0, T[\times \Omega)} \\
& \leq K\|\mathcal{A}\|_{4 a^{\prime} H^{n+2}(] 0, T[\times \Omega)}^{2}\|A\|_{4 a^{\prime} H^{n+2}(] 0, T[\times \Omega)} .
\end{aligned}
$$

We can similarly treat the term $g^{2} C_{a}^{d e} \mathcal{A}^{\mu d}\left(C_{e}^{b c} \mathcal{A}_{\nu c} A_{\mu b}\right)+g^{2} C_{a}^{d e}\left(C_{e}^{b c} \mathcal{A}_{\mu b} \mathcal{A}_{\nu c}\right) A^{\mu d}$ in (7). Moreover:

$\left\|-g C_{a}^{b c} \chi_{\nu c} \partial^{\mu} A_{\mu b}\right\|_{H^{n}(] 0, T[\times \Omega)} \leq K\left\|\chi_{\nu c}\right\|_{4 a^{\prime} H^{n+2}(] 0, T[\times \Omega)} \times\|A\|_{4 a^{\prime} H^{n+2}(] 0, T[\times \Omega)}$ and idem for the term $-2 g C_{a}^{b c} \chi_{\mu b} \partial^{\mu} A_{\nu c}+g C_{a}^{b c} \chi^{\mu b} \partial_{\nu} A_{\mu c}$ in (7). Moreover:

$$
\begin{aligned}
& \left\|g^{2} C_{a}^{d e} \chi^{\mu d}\left(C_{e}^{b c} \mathcal{A}_{\mu b} A_{\nu c}\right)\right\|_{H^{n}(] 0, T[\times \Omega)} \leq K\|\chi\|_{4 a^{\prime} H^{n+2}(] 0, T[\times \Omega)} \\
& \quad \times\|\mathcal{A}\|_{4 a^{\prime} H^{n+2}(] 0, T[\times \Omega)} \times\|A\|_{4 a^{\prime} H^{n+2}(] 0, T[\times \Omega)}
\end{aligned}
$$

and idem for the remaining term in (7) with sometimes $\|\chi\|_{4 a^{\prime} H^{n+2}(10, T[\times \Omega)}$ instead of $\|\mathcal{A}\|_{4 a^{\prime} H^{n+2}(] 0, T[\times \Omega)}$. We finally obtain:

$$
\begin{aligned}
& \left\|d Y M_{w \mathcal{A}} A_{\nu a}\right\|_{H^{n}(] 0, T[\times \Omega)} \leq K\|A\|_{4 a^{\prime} H^{n+2}(] 0, T[\times \Omega)} \\
& \times\left[1+\|\mathcal{A}\|_{4 a^{\prime} H^{n+2}(] 0, T[\times \Omega)}^{2}+\|\chi\|_{4 a^{\prime} H^{n+2}(] 0, T[\times \Omega)}^{2}\right] .
\end{aligned}
$$

So, if we notice as above that $d Y M_{w \mathcal{A}} A$ satisfies the boundary conditions of $F_{n}$ for any $\mathcal{A}$ in $E_{n+2}, d Y M_{w \mathcal{A}}$ is in $L\left(E_{n+2}, F_{n}\right)$. 
Now, is $\mathcal{A} \rightarrow d Y M_{w \mathcal{A}}$ in $C^{0}\left(E_{n+2}, L\left(E_{n+2}, F_{n}\right)\right)$ ? Consider any couple $\left(\mathcal{A}, \mathcal{A}^{\prime}\right)$ in $E_{n+2}^{2}$ and any $A$ in $E_{n+2}$. Denoting $H^{n}(] 0, T[\times \Omega)$ by $H^{n}$, we get:

$$
\begin{aligned}
& \left\|\left(\mathcal{A}_{\mu b}-\mathcal{A}^{\prime}{ }_{\mu b}\right) \partial^{\mu} A_{\nu c}\right\|_{H^{n}}^{2}=\sum_{|\alpha| \leq n}\left\|\partial_{\alpha}\left[\left(\mathcal{A}_{\mu b}-\mathcal{A}^{\prime}{ }_{\mu b}\right) \partial_{\mu} A_{\nu c}\right]\right\|_{L^{2}(] 0, T[\times \Omega)}^{2} \\
& \left\|\left(\mathcal{A}_{\mu b}-\mathcal{A}_{\mu b}^{\prime}\right) \partial^{\mu} A_{\nu c}\right\|_{H^{n}}^{2} \leq \sum_{|\alpha| n \gamma+\delta=\alpha} \sum_{\mid \alpha}\left(\left\|\partial_{\gamma}\left(\mathcal{A}_{\mu b}-\mathcal{A}^{\prime}{ }_{\mu b}\right)\right\|_{L^{4}(] 0, T[\times \Omega)}^{2}\right. \\
& \left.\times\left\|\partial_{\delta} \partial_{\mu} A_{\nu c}\right\|_{L^{4}(] 0, T[\times \Omega)}^{2}\right) .
\end{aligned}
$$

For a constant $C$ independent of $n$, we get:

$$
\begin{aligned}
\left\|\left(\mathcal{A}_{\mu b}-\mathcal{A}^{\prime}{ }_{\mu b}\right) \partial^{\mu} A_{\nu c}\right\|_{H^{n}}^{2} \leq & C \sum_{|\alpha| \leq n \gamma+\delta=\alpha} \sum_{\mid\left\|\partial_{\gamma}\left(\mathcal{A}_{\mu b}-\mathcal{A}^{\prime}{ }_{\mu b}\right)\right\|_{H^{1}(] 0, T[\times \Omega)}^{2}} \\
& \left.\times\left\|\partial_{\delta} \partial_{\mu} A_{\nu c}\right\|_{H^{1}(] 0, T[\times \Omega)}^{2}\right) .
\end{aligned}
$$

By a rough majoration, with $C^{\prime}$ independent of $n$ :

$$
\begin{gathered}
\left\|\left(\mathcal{A}_{\mu b}-\mathcal{A}^{\prime}{ }_{\mu b}\right) \partial^{\mu} A_{\nu c}\right\|_{H^{n}}^{2} \leq C^{\prime}\left\|\mathcal{A}_{\mu b}-\mathcal{A}^{\prime}{ }_{\mu b}\right\|_{H^{n+1}(] 0, T[\times \Omega)}^{2} \\
\left\|\partial_{\mu} A_{\nu c}\right\|_{H^{n+1}(] 0, T[\times \Omega)}^{2} .
\end{gathered}
$$

Now:

$\left\|\left(\mathcal{A}_{\mu b}-\mathcal{A}^{\prime}{ }_{\mu b}\right) \partial^{\mu} A_{\nu c}\right\|_{H^{n}} \leq \sqrt{C^{\prime}}\left\|\mathcal{A}_{\mu b}-\mathcal{A}^{\prime}{ }_{\mu b}\right\|_{H^{n+2}(] 0, T[\times \Omega)}\left\|A_{\nu c}\right\|_{H^{n+2}(] 0, T[\times \Omega)}$.

An analogous calculation affords:

$$
\begin{aligned}
& \|-2 g C_{a}^{b c} \mathcal{A}_{\mu b} \partial^{\mu} A_{\nu c}-g C_{a}^{b c}\left(\partial^{\mu} \mathcal{A}_{\mu b}\right) A_{\nu c}-g C_{a}^{b c} \mathcal{A}_{\nu c} \partial^{\mu} A_{\mu b} \\
& -2 g C_{a}^{b c}\left(\partial^{\mu} \mathcal{A}_{\nu c}\right) A_{\mu b}+g C_{a}^{b c} \mathcal{A}^{\mu b} \partial_{\nu} A_{\mu c}+g C_{a}^{b c}\left(\partial_{\nu} \mathcal{A}_{\mu c}\right) A^{\mu b} \\
& +2 g C^{b c} \mathcal{A}_{\mu b}^{\prime} A_{\nu c}+g C_{a}^{b c}\left(\partial^{\mu} \mathcal{A}^{\prime}{ }_{\mu b}\right) A_{\nu c}+g C_{a}^{b c} \mathcal{A}^{\prime}{ }_{\nu c} \partial^{\mu} A_{\mu b} \\
& +2 g C_{a}^{b c}\left(\partial^{\mu} \mathcal{A}_{\nu c}^{\prime}\right) A_{\mu b}-g C_{a}^{b c} \mathcal{A}^{\prime \mu b} \partial_{\nu} A_{\mu c}-g C_{a}^{b c}\left(\partial_{\nu} \mathcal{A}^{\prime}{ }_{\mu c}\right) A^{\mu b} \|_{H^{n}(] 0, T[\times \Omega)} \\
& \leq C_{1}\left\|\mathcal{A}-\mathcal{A}^{\prime}\right\|_{4 a^{\prime} H^{n+2}(] 0, T[\times \Omega)}\|A\|_{4 a^{\prime} H^{n+2}(] 0, T[\times \Omega)}
\end{aligned}
$$

where $C_{1}$ denotes a constant independent of $n$. Now, with $H^{n}$ written instead of $H^{n}(] 0, T[\times \Omega)$

$$
\begin{aligned}
& \left\|\mathcal{A}_{\mu d} \mathcal{A}_{\mu b} \mathcal{A}_{\nu c}-\mathcal{A}^{\prime}{ }_{\mu d} \mathcal{A}^{\prime}{ }_{\mu b} \mathcal{A}_{\nu c}\right\|_{H^{n}}^{2}=\|\left(\mathcal{A}_{\mu d}-\mathcal{A}^{\prime}{ }_{\mu d}\right) \mathcal{A}_{\mu b} A_{\nu c} \\
& +\left(\mathcal{A}_{\mu b}-\mathcal{A}^{\prime}{ }_{\mu b}\right) \mathcal{A}^{\prime}{ }_{\mu d} A_{\nu c} \|_{H^{n}}^{2} .
\end{aligned}
$$

Therefore:

$$
\begin{aligned}
& \left\|\left(\mathcal{A}_{\mu d}-\mathcal{A}_{\mu d}^{\prime}\right) \mathcal{A}_{\mu b} A_{\nu c}\right\|_{H^{n}}^{2}=\sum_{|\alpha| \leq n}\left\|\partial_{\alpha}\left[\left(\mathcal{A}_{\mu d}-\mathcal{A}^{\prime}{ }_{\mu d}\right) \mathcal{A}_{\mu b} A_{\nu c}\right]\right\|_{L^{2}(] 0, T[\times \Omega)}^{2} \\
& \left\|\left(\mathcal{A}_{\mu d}-\mathcal{A}^{\prime}{ }_{\mu d}\right) \mathcal{A}_{\mu b} A_{\nu c}\right\|_{H^{n}}^{2} \\
& \leq \sum_{|\alpha| \leq n \gamma+\delta=\alpha} \sum_{\kappa+\tau=\delta}\left\|\partial_{\gamma}\left(\mathcal{A}_{\mu d}-\mathcal{A}^{\prime}{ }_{\mu d}\right)\right\|_{L^{8}(] 0, T[\times \Omega)}^{2} \times\left\|\partial_{\kappa} \mathcal{A}_{\mu b}\right\|_{L^{8}(] 0, T[\times \Omega)}^{2} \\
& \times\left\|\partial_{\tau} \mathcal{A}_{\nu c}\right\|_{L^{4}(] 0, T[\times \Omega)}^{2} .
\end{aligned}
$$


So, there exists a constant $C$ independent of $n$ such that:

$$
\begin{aligned}
& \left\|\left(\mathcal{A}_{\mu d}-\mathcal{A}^{\prime}{ }_{\mu d}\right) \mathcal{A}_{\mu b} A_{\nu c}\right\|_{H^{n}}^{2} \leq \\
& C \sum_{\substack{|\alpha| \leq n \gamma+\delta=\alpha\\
}} \sum_{\kappa+\tau=\delta}\left\|\partial_{\gamma}\left(\mathcal{A}_{\mu d}-\mathcal{A}^{\prime}{ }_{\mu d}\right)\right\|_{H^{2}(] 0, T[\times \Omega)}^{2} \times\left\|\partial_{\kappa} \mathcal{A}_{\mu b}\right\|_{H^{2}(] 0, T[\times \Omega)}^{2} \\
& \times\left\|\partial_{\tau} A_{\nu c}\right\|_{H^{1}(] 0, T[\times \Omega)}^{2} .
\end{aligned}
$$

We get by rough majorations for $C^{\prime}$ independent of $n$ :

$$
\begin{aligned}
& \left\|\left(\mathcal{A}_{\mu d}-\mathcal{A}^{\prime}{ }_{\mu d}\right) \mathcal{A}_{\mu b} A_{\nu c}\right\|_{H^{n}} \leq C^{\prime}\left\|\mathcal{A}_{\mu d}-\mathcal{A}^{\prime}{ }_{\mu d}\right\|_{H^{n+2}(] 0, T[\times \Omega)} \\
& \quad \times\left\|\mathcal{A}_{\mu b}\right\|_{H^{n+2}(] 0, T[\times \Omega)} \times\left\|A_{\nu c}\right\|_{H^{n+2}(] 0, T[\times \Omega)} .
\end{aligned}
$$

An analogous majoration brings:

$$
\begin{aligned}
& \| g^{2} C_{a}^{d e} \mathcal{A}^{\mu d}\left(C_{e}^{b c} \mathcal{A}_{\mu b} A_{\nu c}\right)+g^{2} C_{a}^{d e} \mathcal{A}^{\mu d}\left(C_{e}^{b c} A_{\mu b} \mathcal{A}_{\nu c}\right) \\
& +g^{2} C_{a}^{d e}\left(C_{e}^{b c} \mathcal{A}_{\mu b} \mathcal{A}_{\nu c}\right) A^{\mu d}-g^{2} C_{a}^{d e} \mathcal{A}^{\prime \mu d}\left(C_{e}^{b c} \mathcal{A}^{\prime}{ }_{\mu b} A_{\nu c}\right) \\
& -g^{2} C_{a}^{d e} \mathcal{A}^{\prime \mu d}\left(C_{e}^{b d} \mathcal{A}_{\mu b} \mathcal{A}^{\prime}{ }_{\nu c}\right)-g^{2} C_{a}^{d e}\left(C_{a}^{b c} \mathcal{A}^{\prime}{ }_{\mu b} \mathcal{A}^{\prime}{ }_{\nu c}\right) A^{\mu d} \|_{H^{n}(] 0, T[\times \Omega)} \\
& \leq C_{2}\left\|\mathcal{A}-\mathcal{A}^{\prime}\right\|_{4 a^{\prime} H^{n+2}(] 0, T[\times \Omega)} \\
& \times\left(\|\mathcal{A}\|_{4 a^{\prime} H^{n+2}(] 0, T[\times \Omega)}+\left\|\mathcal{A}^{\prime}\right\|_{4 a^{\prime} H^{n+2}(] 0, T[\times \Omega)}\right)\|A\|_{4 a^{\prime} H^{n+2}(] 0, T[\times \Omega)},
\end{aligned}
$$

with $C_{2}$ independent of $n$. Moreover, we get:

$$
\begin{aligned}
& \left\|g^{2} C_{a}^{d e} \chi^{\mu d}\left(C_{e}^{b c}\left(\mathcal{A}_{\mu b}-\mathcal{A}^{\prime}{ }_{\mu b}\right) A_{\nu c}\right)\right\|_{H^{n}} \leq C_{2}^{\prime}\left\|\mathcal{A}-\mathcal{A}^{\prime}\right\|_{4 a^{\prime} H^{n+2}(] 0, T[\times \Omega)} \\
& \quad \times\|\chi\|_{4 a^{\prime} H^{n+2}(] 0, T[\times \Omega)} \times\|A\|_{4 a^{\prime} H^{n+2}(] 0, T[\times \Omega)},
\end{aligned}
$$

with $C_{2}^{\prime}$ independent of $n$ and idem for the other lasting terms of the difference $d Y M_{w \mathcal{A}}-d Y M_{w \mathcal{A}^{\prime}}$ build on the (7) formula. So we get for a constant $C$ independent of $n \geq 0$ (with $C$ obviously $\neq 0$ ):

(9) $\quad\left\|d Y M_{w \mathcal{A}}-d Y M_{w \mathcal{A}^{\prime}}\right\|_{L\left(4 a^{\prime} H_{3}^{n+2}(] 0, T[\times \Omega), 4 a^{\prime} H_{1}^{n}(] 0, T[\times \Omega)\right)} \leq$

$$
\begin{aligned}
& C\left\|\mathcal{A}-\mathcal{A}^{\prime}\right\|_{4 a^{\prime} H_{3}^{n+2}(] 0, T[\times \Omega)}\left(\|\chi\|_{4 a^{\prime} H_{3}^{n+2}(] 0, T[\times \Omega)}+\|\mathcal{A}\|_{4 a^{\prime} H_{3}^{n+2}(] 0, T[\times \Omega)}\right. \\
& \left.+\left\|\mathcal{A}^{\prime}\right\|_{4 a^{\prime} H_{3}^{n+2}(] 0, T[\times \Omega)}\right) .
\end{aligned}
$$

So, $x \rightarrow D f(x)$ is in $C^{0}\left(E_{n+2}, L\left(E_{n+2}, F_{n}\right)\right)$ and the estimate of assumption 5 is fulfilled too.

3.2.5. Assumption 4: tesseralization. For a problem of transcription, we are going to change the exponent of $H^{n}$ into $H^{k}$ and the index $n$ of $E_{n}$ and $F_{n}$ into $k$. Consider the problem $(\mathrm{P})$ :

"for any $F$ in $F_{n}$ and any $\mathcal{A}$ in $E_{n+2}$, to find $A$ in $E_{n+1}$ such that:

$$
d Y M_{w \mathcal{A}} A=F . "
$$

We are going to show that problem $(\mathrm{P})$ has one and only one solution.

We consider the following manner to express any natural integer $\geq 1$ :

$$
\left\{\begin{array}{c}
n=n_{0} a^{\prime}+n_{1}, \quad \text { if } n \leq 4 a^{\prime} \\
n=4 a^{\prime}+4 \times 4 a^{\prime}+4^{2} \times 4 a^{\prime}+\ldots+4^{k(n)} \times 4 a^{\prime} \\
\quad+n_{0} \times 4^{k(n)} \times 4 a^{\prime}+n_{1} \quad \text { if } n>4 a^{\prime}
\end{array}\right.
$$


with:

$$
\left\{\begin{array}{l}
0 \leq n_{0} \leq 3 \\
1 \leq n_{1} \leq 4 a^{\prime} \times 4^{k(n)}
\end{array}\right.
$$

and with the following rules: in the case $n_{1}=4 a^{\prime} \times 4^{k(n)}$, we suppose:

$$
n=4 a^{\prime}+4 \times 4 a^{\prime}+\ldots+4^{k(n)} \times 4 a^{\prime}+n_{0} \times 4 a^{\prime} \times 4^{k(n)}+n_{1}
$$

and not:

$$
n=4 a^{\prime}+\ldots+\left(n_{0}+1\right) \times 4 a^{\prime} \times 4^{k(n)}
$$

if $n_{0} \leq 2$, or:

$$
n=4 a^{\prime}+\ldots+4^{k(n)+1} \times 4 a^{\prime}
$$

if $n_{0}=3$.

If $n \leq 4 a^{\prime}$, we put:

$$
k(n)=-1 .
$$

Let's put:

$$
N_{k(n)}=4 a^{\prime}+4 \times 4 a^{\prime}+\ldots+4^{k(n)} \times 4 a^{\prime}=\left(4^{k(n)+1}-1\right) \times \frac{4}{3} a^{\prime}, \quad k(n) \geq-1 .
$$

So:

$$
N_{-1}=0, N_{0}=4 a^{\prime}, N_{1}=20 a^{\prime}, N_{2}=84 a^{\prime}, \ldots
$$

Therefore:

$$
n=N_{k(n)}+n_{0} \times 4 a^{\prime} \times 4^{k(n)}+n_{1} .
$$

Let's put too:

$$
n^{\prime}=N_{k(n)-1}+n_{1} .
$$

We now understand $N_{k}$ as the number $N_{k(n)}$ with $k(n)=k, k$ being any integer.

For the moment, we consider the finite sequences $u=\left(u_{n}\right)_{1 \leq n \leq N_{k}}$ and $f=$ $\left(f_{n}\right)_{1 \leq n \leq 20 a^{\prime}}$ defined by:

$$
\left\{\begin{array}{l}
u_{\nu+4(a-1)+1}=A_{\nu a}, \quad 1 \leq a \leq a^{\prime}, 0 \leq \nu \leq 3 \\
u_{n}=\partial_{n_{0}} u_{n^{\prime}} \quad \text { for } 4 a^{\prime}<n \leq 20 a^{\prime} \\
u_{n}=\partial_{\left(n^{\prime}\right)_{0}} u_{n^{\prime}+\left(n_{0}-\left(n^{\prime}\right)_{0}\right) \times 4^{k(n)-1} \times 4 a^{\prime}}, \quad \text { for } 20 a^{\prime}<n \leq N_{k}
\end{array}\right.
$$

and:

$$
\left\{\begin{array}{l}
f_{n}=0 \quad \text { for } 1 \leq n \leq 4 a^{\prime} \\
f_{\nu+4(a-1)+1+4 a^{\prime}}=-F_{\nu a} \quad 1 \leq a \leq a^{\prime}, 0 \leq \nu \leq 3 \\
f_{n}=0 \text { for } 8 a^{\prime}<n \leq 20 a^{\prime}
\end{array} .\right.
$$

We consider the $20 a^{\prime} \times 20 a^{\prime}$ matrices $\left(A_{j}^{20 a^{\prime}}\right)$ for $j=1,2,3$ defined by:

$$
\begin{aligned}
& A_{1}^{20 a^{\prime}}=\left(\begin{array}{lllll}
0_{4 a^{\prime}} & 0_{4 a^{\prime}} & 0_{4 a^{\prime}} & 0_{4 a^{\prime}} & 0_{4 a^{\prime}} \\
0_{4 a^{\prime}} & 0_{4 a^{\prime}} & -I d_{4 a^{\prime}} & 0_{4 a^{\prime}} & 0_{4 a^{\prime}} \\
0_{4 a^{\prime}} & -I d_{4 a^{\prime}} & 0_{4 a^{\prime}} & 0_{4 a^{\prime}} & 0_{4 a^{\prime}} \\
0_{4 a^{\prime}} & 0_{4 a^{\prime}} & 0_{4 a^{\prime}} & 0_{4 a^{\prime}} & 0_{4 a^{\prime}} \\
0_{4 a^{\prime}} & 0_{4 a^{\prime}} & 0_{4 a^{\prime}} & 0_{4 a^{\prime}} & 0_{4 a^{\prime}}
\end{array}\right), \\
& A_{2}^{20 a^{\prime}}=\left(\begin{array}{lllll}
0_{4 a^{\prime}} & 0_{4 a^{\prime}} & 0_{4 a^{\prime}} & 0_{4 a^{\prime}} & 0_{4 a^{\prime}} \\
0_{4 a^{\prime}} & 0_{4 a^{\prime}} & 0_{4 a^{\prime}} & -I d_{4 a^{\prime}} & 0_{4 a^{\prime}} \\
0_{4 a^{\prime}} & 0_{4 a^{\prime}} & 0_{4 a^{\prime}} & 0_{4 a^{\prime}} & 0_{4 a^{\prime}} \\
0_{4 a^{\prime}} & -I d_{4 a^{\prime}} & 0_{4 a^{\prime}} & 0_{4 a^{\prime}} & 0_{4 a^{\prime}} \\
0_{4 a^{\prime}} & 0_{4 a^{\prime}} & 0_{4 a^{\prime}} & 0_{4 a^{\prime}} & 0_{4 a^{\prime}}
\end{array}\right),
\end{aligned}
$$




$$
A_{3}^{20 a^{\prime}}=\left(\begin{array}{lllll}
0_{4 a^{\prime}} & 0_{4 a^{\prime}} & 0_{4 a^{\prime}} & 0_{4 a^{\prime}} & 0_{4 a^{\prime}} \\
0_{4 a^{\prime}} & 0_{4 a^{\prime}} & 0_{4 a^{\prime}} & 0_{4 a^{\prime}} & -I d_{4 a^{\prime}} \\
0_{4 a^{\prime}} & 0_{4 a^{\prime}} & 0_{4 a^{\prime}} & 0_{4 a^{\prime}} & 0_{4 a^{\prime}} \\
0_{4 a^{\prime}} & 0_{4 a^{\prime}} & 0_{4 a^{\prime}} & 0_{4 a^{\prime}} & 0_{4 a^{\prime}} \\
0_{4 a^{\prime}} & -I d_{4 a^{\prime}} & 0_{4 a^{\prime}} & 0_{4 a^{\prime}} & 0_{4 a^{\prime}}
\end{array}\right),
$$

where $0_{4 a^{\prime}}$ denotes the null $4 a^{\prime} \times 4 a^{\prime}$ matrix and $I d_{4 a^{\prime}}$ denotes the $4 a^{\prime} \times 4 a^{\prime}$ identity matrix. So, we consider the $20 a^{\prime} \times 20 a^{\prime}$ system given by:

$$
M u=\partial_{0} u+\sum_{j=1}^{3} A_{j}^{20 a^{\prime}} \partial_{j} u+B^{20 a^{\prime}}(x) u=f,
$$

where the $20 a^{\prime} \times 20 a^{\prime} B^{20 a^{\prime}}(x)$ matrix is composed by $-I d_{4 a^{\prime}}$ for the second block of its first line of $4 a^{\prime} \times 4 a^{\prime}$ blocks, and $0_{4 a^{\prime}}$ for the other blocks of its first line of $4 a^{\prime} \times 4 a^{\prime}$ blocks. Notice that we have to write $-F_{\nu a}$ and not $F_{\nu a}$ in the right member of the second equation in (11) since we have chosen $\partial_{0} u$ and not $-\partial_{0} u$ in (12). Moreover, $B^{20 a^{\prime}}(x)$ is build to make the $n^{t h}$ line of $M u=f$ for $4 a^{\prime}<n \leq 8 a^{\prime}$ equal to:

$$
d Y M_{w \mathcal{A}} u_{n}=f_{n}
$$

and with only $0 \mathrm{~s}$ on the lines of rank $n>8 a^{\prime}$.

Now, starting from the $20 a^{\prime} \times 20 a^{\prime}$ system (12), we can build a $N_{k} \times N_{k}$ system (see (17) below): indeed, if we denote by $l_{n}$ the $n^{\text {th }}$ of (17), we form (17) by:

$$
l_{n}=\partial_{\left(n^{\prime}\right)_{0}} l_{n^{\prime}+\left(n_{0}-\left(n^{\prime}\right)_{0}\right) \times 4^{k(n)-1} \times 4 a^{\prime}}, \quad \text { for } 20 a^{\prime}<n \leq N_{k} .
$$

We call the process a "tesseralization". In fact, the order of derivation of $u_{n}$ in (10) brings by a permutation of the derivatives:

$$
\begin{aligned}
& \left(u_{n}\right)=\left(u_{1}, \ldots, u_{4 a^{\prime}}, \partial_{0} u_{1}, \ldots, \partial_{0} u_{4 a^{\prime}}, \partial_{1} u_{1}, \ldots, \partial_{1} u_{4 a^{\prime}}, \partial_{2} u_{1}, \ldots, \partial_{2} u_{4 a^{\prime}},\right. \\
& \partial_{3} u_{1}, \ldots, \partial_{3} u_{4 a^{\prime}}, \partial_{0} \partial_{0} u_{1}, \partial_{0} \partial_{0} u_{2}, \ldots, \partial_{0} \partial_{3} u_{4 a^{\prime}}, \partial_{1} \partial_{0} u_{1}, \ldots, \partial_{1} \partial_{3} u_{4 a^{\prime}}, \\
& \partial_{2} \partial_{0} u_{1}, \ldots, \partial_{2} \partial_{3} u_{4 a^{\prime}}, \partial_{3} \partial_{0} u_{1}, \ldots, \partial_{3} \partial_{3} u_{4 a^{\prime}}, \partial_{0} \partial_{0} \partial_{0} u_{1} \\
& \left.\partial_{0} \partial_{0} \partial_{0} u_{2}, \ldots, \partial_{3}^{k} u_{4 a^{\prime}}\right)
\end{aligned}
$$

and so brings the natural order of derivation on $u_{n}$ in (10):

$$
u_{n}=\partial_{n_{0}} u_{n^{\prime}} \text { for } 4 a^{\prime}<n \leq N_{k} .
$$

So, we can consider the sequence $\left(u_{n}\right)_{1 \leq n \leq N_{k}}$ defined by $(10)$ and $\left(f_{n}\right)_{1 \leq n \leq N_{k}}$ by (11) and:

$$
f_{n}=\partial_{\left(n^{\prime}\right)_{0}} f_{n^{\prime}+\left(n_{0}-\left(n^{\prime}\right)_{0}\right) \times 4^{k(n)-1} \times 4 a^{\prime}}, \quad \text { for } 20 a^{\prime}<n \leq N_{k} .
$$

So, we obtain a $N_{k} \times N_{k}$ system (17) by this process of "tesseralization" of system (12) from order $20 a^{\prime}+1$ to order $N_{k}$ :

$$
L u=\partial_{0} u+\sum_{j=1}^{3} A_{j} \partial_{j} u+B(x) u=f_{n} .
$$

The $A_{1}$ matrix (which has not to be confused with the $A_{1}$ component of the gauge field $A$ ), is composed on its main diagonal by the first block (top-left) $A_{1}^{20 a^{\prime}}$ and 
then on the remaining part of its main diagonal, by a finite sequence of blocks for $n=1$ to $k-1$ :

$$
\left(\begin{array}{llll}
0_{4^{n} \times 4 a^{\prime}} & -I d_{4^{n} \times 4 a^{\prime}} & 0_{4^{n} \times 4 a^{\prime}} & 0_{4^{n} \times 4 a^{\prime}} \\
-I d_{4^{n} \times 4 a^{\prime}} & 0_{4^{n} \times 4 a^{\prime}} & 0_{4^{n} \times 4 a^{\prime}} & 0_{4^{n} \times 4 a^{\prime}} \\
0_{4^{n} \times 4 a^{\prime}} & 0_{4^{n} \times 4 a^{\prime}} & 0_{4^{n} \times 4 a^{\prime}} & 0_{4^{n} \times 4 a^{\prime}} \\
0_{4^{n} \times 4 a^{\prime}} & 0_{4^{n} \times 4 a^{\prime}} & 0_{4^{n} \times 4 a^{\prime}} & 0_{4^{n} \times 4 a^{\prime}}
\end{array}\right)
$$

and $0 \mathrm{~s}$ anywhere else. The $A_{2}$ matrix is composed ont its principal diagonal by the first block (top-left) $A_{2}^{20 a^{\prime}}$ and then on the remaining part of its principal diagonal, by a finite sequence of blocks for $n=1$ to $k-1$ :

$$
\left(\begin{array}{llll}
0_{4^{n}} \times 4 a^{\prime} & 0_{4^{n} \times 4 a^{\prime}} & -I d_{4^{n} \times 4 a^{\prime}} & 0_{4^{n} \times 4 a^{\prime}} \\
0_{4^{n}} \times 4 a^{\prime} & 0_{4^{n}} \times 4 a^{\prime} & 0_{4^{n} \times 4 a^{\prime}} & 0_{4^{n} \times 4 a^{\prime}} \\
-I d_{4^{n} \times 4 a^{\prime}} & 0_{4^{n} \times 4 a^{\prime}} & 0_{4^{n} \times 4 a^{\prime}} & 0_{4^{n} \times 4 a^{\prime}} \\
0_{4^{n} \times 4 a^{\prime}} & 0_{4^{n} \times 4 a^{\prime}} & 0_{4^{n} \times 4 a^{\prime}} & 0_{4^{n} \times 4 a^{\prime}}
\end{array}\right)
$$

and $0 s$ anywhere else. The $A_{3}$ matrix is composed on its main diagonal by the first block (top-left) $A_{3}^{20 a^{\prime}}$ and then on the remaining part of its main diagonal, by a finite sequence of blocks for $n=1$ to $k-1$ :

$$
\left(\begin{array}{llll}
0_{4^{n} \times 4 a^{\prime}} & 0_{4^{n} \times 4 a^{\prime}} & 0_{4^{n} \times 4 a^{\prime}} & -I d_{4^{n} \times 4 a^{\prime}} \\
0_{4^{n} \times 4 a^{\prime}} & 0_{4^{n} \times 4 a^{\prime}} & 0_{4^{n} \times 4 a^{\prime}} & 0_{4^{n} \times 4 a^{\prime}} \\
0_{4^{n} \times 4 a^{\prime}} & 0_{4^{n} \times 4 a^{\prime}} & 0_{4^{n} \times 4 a^{\prime}} & 0_{4^{n} \times 4 a^{\prime}} \\
-I d_{4^{n} \times 4 a^{\prime}} & 0_{4^{n} \times 4 a^{\prime}} & 0_{4^{n} \times 4 a^{\prime}} & 0_{4^{n} \times 4 a^{\prime}}
\end{array}\right)
$$

and 0 s anywhere else. The important point for the possibility to apply the finite propagation speed method is that $A_{1}, A_{2}$ and $A_{3}$ are symmetrical. So, for $n>4 a^{\prime}$ with $n_{0} \neq 0$, then the $n^{\text {th }}$ line of $B(x)$ is only composed by 0 s and:

$$
f_{n}=0
$$

too.

3.2.6. Assumption 4: finite propagation speed. Now, we consider $\mathcal{O}(s)=$ ] $0, s[\times \Omega$ and we put:

$$
\Sigma_{t}=\{t\} \times \Omega .
$$

We consider the Hermitian product $(,)_{s}$ in ${ }^{N_{k}} L^{2}(\mathcal{O}(s))$ for $k \geq 7$. We put:

$$
\mathcal{O}=\mathcal{O}(T) \text {. }
$$

We obtain for any $u$ smooth in $[0, T] \times \mathbb{R}_{1} \times \mathbb{R}_{2} \times \mathbb{R}_{3}$ periodic in $x_{1}, x_{2}, x_{3}$ with the period $2 N$ ( $N$ presented in paragraph 3.1$)$ :

$$
(L u, u)_{s}+(u, L u)_{s}-(u, B u)_{s}-(B u, u)_{s}=\int_{\Sigma_{s}}|u|^{2} d S-\int_{\Sigma_{0}}|u|^{2} d S .
$$

Notice that (19) is equivalent to formula (5.22) [5,volume 1,page 437]. So:

$$
2 \operatorname{Re}(L u, u)_{s}-2 \operatorname{Re}(B u, u)_{s}=\int_{\Sigma_{s}}|u|^{2} d S-\int_{\Sigma_{0}}|u|^{2} d S .
$$

But, if we do rather the same calculations as for the proof of assumptions 2 and 5, using the following inclusion:

$$
H^{3}\left(\mathbb{R}^{4}\right) \subset_{c} L^{\infty}\left(\mathbb{R}^{4}\right)
$$


we get:

$$
\begin{aligned}
& \int_{\mathcal{O}(s)}|B u|^{2} d t \\
& \leq C\left(\|\mathcal{A}\|_{\mathcal{O}(s)}^{4}+\|\chi\|_{\mathcal{O}(s)}^{4}+1\right) \int_{\mathcal{O}(s)}|u|^{2} d t,
\end{aligned}
$$

where the constant $C$ is independent of the order $k$ of the Sobolev spaces.

So, if we put:

$$
E(s)=\|u\|_{N_{k} L^{2}(\mathcal{O}(s))}^{2},
$$

we get:

$$
\frac{d E}{d s}(s) \leq 2 \int_{\mathcal{O}(s)}|L u \times \bar{u}| d V+2 \int_{\mathcal{O}(s)}|B u \times \bar{u}| d V+\int_{\Sigma_{0}}|u|^{2} d S .
$$

Suppose that:

$$
\partial_{0}^{m} u_{n}(0, x)=0 \quad \text { for } 1 \leq n \leq 4 a^{\prime}, \quad \text { for } m=0,1,
$$

then we obtain for $\alpha=\left(\alpha_{0}, \alpha_{1}, \alpha_{2}, \alpha_{3}\right)$ with $\left|\left(\alpha_{1}, \alpha_{2}, \alpha_{3}\right)\right| \neq 0$ :

$$
\partial^{\alpha} u_{n}(0, x)=0 \text { for any } n \text {. }
$$

Now, we suppose that problem (P) has a solution for $u$ and $F$ smooth. Using:

$$
\partial_{0}^{2} u_{n}-\Delta u_{n}+B_{n+4 a^{\prime}}(u)=-f_{n+4 a^{\prime}} \text { for } 1 \leq n \leq 4 a^{\prime},
$$

where $B_{n}(u)$ expresses the $n^{\text {th }}$ line of $B u$, we obtain by induction:

$$
\partial_{0}^{m+2} u_{n}(0, x)=-\partial_{0}^{m} f_{n+4 a^{\prime}}(0, x)-\partial_{0}^{m} B_{n+4 a^{\prime}}(u)(0, x),
$$

for $1 \leq n \leq 4 a^{\prime}$. Therefore:

$$
\begin{aligned}
\int_{\Sigma_{0}}|u|^{2} d S= & \sum_{j=0}^{k-2} \sum_{n=1}^{4 a^{\prime}} \int_{\Sigma_{0}}\left|\partial_{0}^{j} f_{n+4 a^{\prime}}(0, x)\right|^{2} d S \\
& +\sum_{j=0}^{k-2} \sum_{n=1}^{4 a^{\prime}} \int_{\Sigma_{0}}\left|\partial_{0}^{j} B_{n+4 a^{\prime}}(u)(0, x)\right|^{2} d S .
\end{aligned}
$$

Now, we use the continuous trace map from $H^{1}(\mathcal{O}(s))$ into $L^{2}\left(\Sigma_{0}\right)$ to get:

$$
\begin{aligned}
& \text { (21) } \int_{\Sigma_{0}}|u|^{2} d S \leq K \sum_{i=0}^{k-2} \sum_{j=0}^{3} \sum_{n=1}^{4 a^{\prime}} \int_{\mathcal{O}(s)}\left|\partial_{0}^{i} \partial_{j} f_{n+4 a^{\prime}}\right|^{2} d V \\
& +K \sum_{n=0}^{k-2} \sum_{n=1}^{4 a^{\prime}} \int_{\mathcal{O}(s)}\left|\partial_{0}^{i} f_{n+4 a^{\prime}}\right|^{2} d V+K \sum_{i=0}^{k-2} \sum_{j=0}^{3} \sum_{n=1}^{4 a^{\prime}} \int_{\mathcal{O}(s)}\left|\partial_{0}^{i} \partial_{j} B_{n+4 a^{\prime}}(u)\right|^{2} d V \\
& +K \sum_{i=0}^{k-2} \sum_{n=1}^{4 a^{\prime}} \int_{\mathcal{O}(s)}\left|\partial_{0}^{i} B_{n+4 a^{\prime}}(u)\right|^{2} d V .
\end{aligned}
$$

Therefore, we get for a constant $K^{\prime}$ independent of $k$ :

$$
\int_{\Sigma_{0}}|u|^{2} d S \leq K^{\prime}\|L u\|_{N_{k} L^{2}(\mathcal{O}(s))}^{2}+K^{\prime}\|B u\|_{N_{k} L^{2}(\mathcal{O}(s))}^{2} .
$$


So:

$$
\int_{\Sigma_{0}}|u|^{2} d S \leq\left(C_{1}\left(\|\mathcal{A}\|_{4 a^{\prime} H^{k+2}(\mathcal{O}(s))}^{4}+\|\chi\|_{4 a^{\prime} H^{k+2}(\mathcal{O}(s))}^{4}\right)+C_{1}\right)
$$

$$
\times\|u\|_{N_{k} L^{2}(\mathcal{O}(s))}^{2}+K^{\prime} \times\|L u\|_{N_{k} L^{2}(\mathcal{O}(s))}^{2},
$$

with constants $K^{\prime}$ and $C_{1}$ independent of $k$. Then, according to (20), we get for any $M^{\prime \prime}>0$ (and $k \geq 7$ ):

$$
\begin{aligned}
& \frac{d E}{d s}(s) \leq\left(\frac{1}{2 M^{\prime \prime}}+K_{1}\right)\|L u\|_{N_{k} L^{2}(\mathcal{O}(s))}^{2} \\
& +\left[2 M^{\prime \prime}+C_{3}\left(\|\mathcal{A}\|_{4 a^{\prime} H^{k+2}(\mathcal{O}(s))}^{4}+\|\chi\|_{4 a^{\prime} H^{k+2}(\mathcal{O}(s))}^{4}\right)+C_{3}\right] \times E(s),
\end{aligned}
$$

with constants $K_{1}$ and $C_{3}$ independent of $k$ and $\mathcal{A}$.

Then, by the Gronwall lemma, we get:

$$
\begin{aligned}
\text { (23) } \quad & E(s) \leq s\left(\frac{1}{2 M^{\prime \prime}}+K_{1}\right)\|L u\|_{N_{k} L^{2}(\mathcal{O}(s))}^{2} \times \\
& e^{\int}\left[C_{3}\left(\|\mathcal{A}\|_{4 a^{\prime} H^{k+2}(\mathcal{O}(\sigma))}^{4}+\|\chi\|_{4 a^{\prime} H^{k+2}(\mathcal{O}(\sigma))}^{4}\right)+C_{3}+2 M^{\prime \prime}\right] d \sigma .
\end{aligned}
$$

So:

$$
\|u\|_{N_{k} L^{2}(\mathcal{O})} \leq C_{\mathcal{A}, k}\|L u\|_{N_{k} L^{2}(\mathcal{O})},
$$

with a constant $C_{\mathcal{A}, k}$ depending on $k$ and $\mathcal{A}$. Now, we are going to show that (24) occurs for:

$$
C_{\mathcal{A}, k}=k_{0} \sqrt{T} \times\left(\|\mathcal{A}\|_{4 a^{\prime} H^{k+2}(\mathcal{O})}^{4}+\|\chi\|_{4 a^{\prime} H^{k+2}(\mathcal{O})}^{4}\right)
$$

where the constant $k_{0}$ is independent of $\mathcal{A}, T$ and of the order $k$ of the Sobolev spaces.

So, if we consider the constants $C_{3}$ in (23) and $s$ such that:

$$
\left[2 M^{\prime \prime}+C_{3}\left(\|\mathcal{A}\|_{4 a^{\prime} H^{k+2}(\mathcal{O})}^{4}+\|\mathcal{A}\|_{4 a^{\prime} H^{k+2}(\mathcal{O})}^{4}\right)+C_{3}\right] s=1,
$$

then, we get:

$$
\|u\|_{N_{k} L^{2}(\mathcal{O}(s))}^{2} \leq e s\left(\frac{1}{2 M^{\prime \prime}}+K_{1}\right)\|L u\|_{N_{k} L^{2}(\mathcal{O}(s))}^{2} .
$$

So, if we share $[0, T]$ in intervals of length $\Delta T$ with:

$$
\left[2 M^{\prime \prime}+C_{3}\left(\|\mathcal{A}\|_{4 a^{\prime} H^{k+2}(\mathcal{O})}^{4}+\|\mathcal{A}\|_{4 a^{\prime} H^{k+2}(\mathcal{O})}^{4}\right)+C_{3}\right] \Delta T=1,
$$

then putting:

$$
\left\{\begin{array}{l}
\left.\mathcal{O}^{n}=\right] n \Delta T,(n+1) \Delta T[\times \Omega, \text { for } 0 \leq n \leq \mathcal{M}, \text { with : } \\
\mathcal{M}=\left[2 M^{\prime \prime}+C_{3}\left(\|\mathcal{A}\|_{4 a^{\prime} H^{k+2}(\mathcal{O})}^{4}+\|\chi\|_{4 a^{\prime} H^{k+2}(\mathcal{O})}^{4}\right)+C_{3}\right] \times T-1
\end{array}\right.
$$

(we suppose $\mathcal{M}$ integer), we get if $u_{n}=0$ for $1 \leq n \leq 4 a^{\prime}$ on $\Sigma_{n \Delta T}=\{n \Delta T\} \times \Omega$ :

$$
\|u\|_{N_{k} L^{2}\left(\mathcal{O}^{n}\right)}^{2} \leq \Delta T e\left(\frac{1}{2 M^{\prime \prime}}+K_{1}\right)\|L u\|_{N_{k} L^{2}\left(\mathcal{O}^{n}\right)}^{2} .
$$

Now, if we denote by $u_{r, n}$ the function such that:

$$
u_{r, n}(n \Delta T+t, x)=u(n \Delta T-t, x), \quad \text { for } 1 \leq n \leq 4 a^{\prime}
$$


and by $L_{r, n}$ the operator obtained from $L$ by transforming all its coefficients in the (27) way, then, by putting:

$$
\frac{1}{M}=\frac{e\left(\frac{1}{2 M^{\prime \prime}}+K_{1}\right)}{2 M^{\prime \prime}+C_{3}\left(\|\mathcal{A}\|_{4 a^{\prime} H^{k+2}(\mathcal{O})}^{4}+\|\chi\|_{4 a^{\prime} H^{k+2}(\mathcal{O})}^{4}\right)+C_{3}}
$$

we get (since $u_{r, n}(n \Delta T, x)-u(n \Delta T, x)=0$ on $\Sigma_{n \Delta T}$ for $\left.1 \leq n \leq 4 a^{\prime}\right)$ :

$$
\left\|u-u_{r, n}\right\|_{N_{k} L^{2}\left(\mathcal{O}^{n}\right)}^{2} \leq \frac{1}{M}\left\|L\left(u-u_{r, n}\right)\right\|_{N_{k} L^{2}\left(\mathcal{O}^{n}\right)}^{2} .
$$

Since $\mathcal{A} \rightarrow d Y M_{w \mathcal{A}}$ is in $C^{0}\left(E_{n+2}, L\left(E_{n+2}, F_{n}\right)\right.$ ), for $\Delta T$ sufficiently small (choosing $C_{3}$ ad hoc to get this), we get:

$$
\left\|L_{r, n} u_{r, n}-L u_{r, n}\right\|_{N_{k} L^{2}\left(\mathcal{O}^{n}\right)}^{2} \leq \frac{K}{M}\|u\|_{N_{k} L^{2}\left(\mathcal{O}^{n-1}\right)}^{2},
$$

where we can put:

$$
K=\frac{1}{e\left(\frac{1}{2 M^{\prime \prime}}+K_{1}\right)} .
$$

We do now ad hoc majorations to obtain the recurrent inequalities (29) (see further beyond):

$$
\begin{aligned}
\left\|u-u_{r, n}\right\|_{N_{k} L^{2}\left(\mathcal{O}^{n}\right)}^{2} \leq & \frac{1}{M}\left\|L\left(u-u_{r, n}\right)\right\|_{N_{k} L^{2}\left(\mathcal{O}^{n}\right)}^{2} \\
\left\|u-u_{r, n}\right\|_{N_{k} L^{2}\left(\mathcal{O}^{n}\right)}^{2} \leq & \frac{2}{M}\|L u\|_{N_{k} L^{2}\left(\mathcal{O}^{n}\right)}^{2}+\frac{4}{M}\left\|L_{r, n} u_{r, n}-L u_{r, n}\right\|_{N_{k} L^{2}\left(\mathcal{O}^{n}\right)}^{2} \\
& +\frac{4}{M}\left\|L_{r, n} u_{r, n}\right\|_{N_{k} L^{2}\left(\mathcal{O}^{n}\right)}^{2} \\
\left\|u-u_{r, n}\right\|_{N_{k} L^{2}\left(\mathcal{O}^{n}\right)}^{2} \leq & \frac{2}{M}\|L u\|_{N_{k} L^{2}\left(\mathcal{O}^{n}\right)}^{2}+\frac{4 K}{M^{2}}\|u\|_{N_{k} L^{2}\left(\mathcal{O}^{n-1}\right)}^{2} \\
& \frac{4}{M}\|L u\|_{N_{k} L^{2}\left(\mathcal{O}^{n-1}\right)}^{2}
\end{aligned}
$$

So:

$$
\begin{aligned}
& \|u\|_{N_{k} L^{2}\left(\mathcal{O}^{n}\right)} \leq \\
& \frac{\sqrt{2}}{\sqrt{M}}\|L u\|_{N_{k} L^{2}\left(\mathcal{O}^{n}\right)}+\frac{2 \sqrt{K}}{M}\|u\|_{N_{k} L^{2}\left(\mathcal{O}^{n-1}\right)}+\frac{2}{\sqrt{M}}\|L u\|_{N_{k} L^{2}\left(\mathcal{O}^{n-1}\right)} \\
& +\left\|u_{r, n}\right\|_{N_{k} L^{2}\left(\mathcal{O}^{n}\right)} \\
& \|u\|_{N_{k} L^{2}\left(\mathcal{O}^{n}\right)} \leq \\
& \frac{\sqrt{2}}{\sqrt{M}}\|L u\|_{N_{k} L^{2}\left(\mathcal{O}^{n}\right)}+\frac{2}{\sqrt{M}}\|L u\|_{N_{k} L^{2}\left(\mathcal{O}^{n-1}\right)} \\
& +\left(\frac{2 \sqrt{K}}{M}+1\right)\|u\|_{N_{k} L^{2}\left(\mathcal{O}^{n-1}\right)} .
\end{aligned}
$$

But we get:

$$
\|u\|_{N_{k} L^{2}\left(\mathcal{O}^{0}\right)} \leq \frac{1}{\sqrt{M}}\|L u\|_{N_{k} L^{2}\left(\mathcal{O}^{0}\right)}
$$

and so:

$$
\|u\|_{N_{k} L^{2}\left(\mathcal{O}^{1}\right)} \leq \frac{\sqrt{2}}{\sqrt{M}}\|L u\|_{N_{k} L^{2}\left(\mathcal{O}^{1}\right)}+\left(\frac{3}{\sqrt{M}}+\frac{2 \sqrt{K}}{M^{\frac{3}{2}}}\right)\|L u\|_{N_{k} L^{2}\left(\mathcal{O}^{0}\right)} .
$$


So, we can obtain a majoration of the type:

$$
\|u\|_{N_{k} L^{2}\left(\mathcal{O}^{n}\right)} \leq \sum_{i=0}^{n} a_{n, i}\|L u\|_{N_{k} L^{2}\left(\mathcal{O}^{i}\right)} .
$$

We put:

Now, we consider:

$$
\left\{\begin{array}{l}
a_{0,0}=\frac{1}{\sqrt{M}} \\
a_{1,0}=\left(\frac{2 \sqrt{K}}{M}+1\right) \times \frac{4}{\sqrt{M}} \\
a_{2,0}=\left(\frac{2 \sqrt{K}}{M}+1\right)^{2} \times \frac{4}{\sqrt{M}} \\
a_{n, 0}=\left(\frac{2 \sqrt{K}}{M}+1\right) a_{n-1,0}, \quad \text { for } n \geq 3
\end{array} .\right.
$$

$$
M^{\prime \prime}=\|\mathcal{A}\|_{4 a^{\prime} H^{k+2}(\mathcal{O})}^{4} .
$$

So, for $\|\mathcal{A}\|_{4 a^{\prime} H^{k+2}(\mathcal{O})}$ sufficiently great, since $M=\frac{\mathcal{M}+1}{\operatorname{Tex}\left(\frac{1}{2 M^{\prime \prime}}+K_{1}\right)}$, we obtain:

$$
\sum_{i=0}^{\mathcal{M}} a_{i, 0}^{2} \leq \frac{1}{M}+\frac{17}{M} \times \frac{1-\left(\frac{2 \sqrt{K}}{M}+1\right)^{2 \mathcal{M}}}{1-\left(\frac{2 \sqrt{K}}{M}+1\right)^{2}} .
$$

Therefore:

$$
\begin{aligned}
& \sum_{i=0}^{\mathcal{M}} a_{i, 0}^{2} \leq \frac{1}{M}+\frac{18}{M} \times \frac{\frac{4 \sqrt{K} \mathcal{M}}{M}}{\frac{4 \sqrt{K}}{M}} \\
& \sum_{i=0}^{\mathcal{M}} a_{i, 0}^{2} \leq \frac{1}{M}+18\left(\frac{1}{2 M^{\prime \prime}}+K_{1}\right) T e \\
& \text { (28) } \quad \sum_{i=0}^{\mathcal{M}} a_{i, 0}^{2} \leq \frac{T e\left(\frac{1}{2 M^{\prime \prime}}+K_{1}\right)}{\mathcal{M}+1}+18\left(\frac{1}{2 M^{\prime \prime}}+K_{1}\right) T e
\end{aligned}
$$

We put:

$$
r=\frac{2 \sqrt{K}}{M}+1 .
$$

We want to get the following recurrent inequalities for $n \geq 1$ :

$$
a_{n, 0} \geq a_{n, 1} \geq \ldots \geq a_{n, n-2} \geq a_{n, n-1} \geq a_{n, n} \text { and } a_{n, n-1} \geq a_{n+1, n} .
$$

Indeed, for $n=1$, we can put:

$$
a_{1,1}=\frac{\sqrt{2}}{\sqrt{M}}
$$

and so:

$$
a_{1,0} \geq a_{1,1} .
$$

For $n=2$, we have put and we can put:

$$
a_{2,0}=r^{2} \times \frac{4}{\sqrt{M}}, \quad a_{2,1}=\frac{2}{\sqrt{M}}+\frac{\sqrt{2}}{\sqrt{M}} \times r, \quad a_{2,2}=\frac{\sqrt{2}}{\sqrt{M}},
$$

so, since $r>1$ :

$$
a_{2,0} \geq a_{2,1} \geq a_{2,2} \quad \text { and } a_{1,0} \geq a_{2,1} .
$$


Moreover:

$$
a_{3,2}=\frac{2}{\sqrt{M}}+r \frac{\sqrt{2}}{\sqrt{M}}
$$

so that:

$$
a_{2,1} \geq a_{3,2} .
$$

So, inequalities (29) are fulfilled for $n=1$ and 2. Suppose that for the rank $n \geq 2$, we get the above recurrent inequalities (29). Then:

$$
\left\{\begin{array}{l}
a_{n+1, n+1}=\frac{\sqrt{2}}{\sqrt{M}} \\
a_{n+1, n}=\frac{2}{\sqrt{M}}+r a_{n, n} \\
a_{n+1, i}=r a_{n, i}, \quad \text { for } 0 \leq i<n
\end{array} .\right.
$$

So, using the inductive inequalities (29) for the rank $n$, we obtain those of rank $n+1$ until $i=n-1$ :

$$
a_{n+1,0} \geq a_{n+1,1} \geq \ldots \geq a_{n+1, n-2} \geq a_{n+1, n-1} .
$$

Moreover:

$$
a_{n+1, n-1}=r a_{n, n-1} \geq r a_{n+1, n} \geq a_{n+1, n} .
$$

Moreover:

$$
\begin{aligned}
& a_{n+2, n+1}=\frac{2}{\sqrt{M}}+r a_{n+1, n+1} \\
& a_{n+2, n+1}=\frac{2}{\sqrt{M}}+\frac{\sqrt{2}}{\sqrt{M}} r \\
& a_{n+2, n+1} \leq \frac{2}{\sqrt{M}}+r a_{n, n}
\end{aligned}
$$

so that:

$$
a_{n+1, n} \geq a_{n+2, n+1} .
$$

Moreover, we evidently get:

$$
a_{n+1, n} \geq a_{n+1, n+1} .
$$

So, we get (29) for the rank $n+1$. Therefore, we get the first set of recurrent inequalities (29) for any rank $n, 0 \leq n \leq \mathcal{M}$.

So, we obtain for $M$ sufficiently great and any $k \geq 7$ :

$$
\sum_{n=0}^{\mathcal{M}}\|u\|_{N_{k} L^{2}\left(\mathcal{O}^{n}\right)}^{2} \leq \sum_{n=0}^{\mathcal{M}}(n+1) \sum_{i=0}^{n} a_{n, i}^{2}\|L u\|_{N_{k} L^{2}\left(\mathcal{O}^{i}\right)}^{2} .
$$

By a rough majoration, using (28) and (29):

$$
\begin{aligned}
\|u\|_{N_{k} L^{2}(\mathcal{O})}^{2} \leq & (\mathcal{M}+1)^{2}\left(\sum_{n=0}^{\mathcal{M}} a_{n, 0}^{2}\right) \times\|L u\|_{N_{k} L^{2}(\mathcal{O})}^{2} \\
\|u\|_{N_{k} L^{2}(\mathcal{O})}^{2} \leq & (\mathcal{M}+1)^{2} \times\left[\frac{T e\left(\frac{1}{2 M^{\prime \prime}}+K_{1}\right)}{\mathcal{M}+1}+18 T e\left(\frac{1}{2 M^{\prime \prime}}+K_{1}\right)\right] \\
& \times\|L u\|_{N_{k} L^{2}(\mathcal{O})}^{2} .
\end{aligned}
$$


So, by a rough majoration:

$$
\begin{aligned}
\|u\|_{N_{k} L^{2}(\mathcal{O})} \leq & k_{0} \sqrt{T} \times\left(\|\mathcal{A}\|_{4 a^{\prime} H^{k+2}(\mathcal{O})}^{4}+\|\chi\|_{4 a^{\prime} H^{k+2}(\mathcal{O})}^{4}+\frac{1}{3}\right) \\
& \times\|L u\|_{N_{k} L^{2}(\mathcal{O})}
\end{aligned}
$$

where $k_{0}$ is independent of $\mathcal{A}, T$ and the Sobolev order $k$. So, we get for any $k \geq 7$ :

$$
\begin{aligned}
\|A\|_{4 a^{\prime} H^{k}(] 0, T[\times \Omega)} \leq & k_{0} \sqrt{T} \times\left(\|\mathcal{A}\|_{4 a^{\prime} H^{k+2}(\mathcal{O})}^{4}+\|\chi\|_{4 a^{\prime} H^{k+2}(\mathcal{O})}^{4}+\frac{1}{3}\right) \\
& \times\left\|d Y M_{w, \mathcal{A}} A\right\|_{4 a^{\prime} H^{k-1}(] 0, T[\times \Omega)} .
\end{aligned}
$$

Now, we have to build the solution to problem $(\mathrm{P})$. We can approximate the coefficients of $L$ by real analytic functions $A_{\nu}=\left(A_{\nu 1}, A_{\nu 2}, A_{\nu 3}\right)$ and $B_{\nu}$ up to the order $20 a^{\prime}$, on $\mathbb{R} \times \Omega$; we can conceive these functions as being defined on $\mathbb{R} \times \mathbb{R}^{3}$ and can arrange their coefficients to have entire holomorphic extension to $\mathbb{C} \times \mathbb{C}^{3}$ with the $2 N$-periodicity in $\left(x_{1}, x_{2}, x_{3}\right)$ (with the $N$ introduced in paragraph 3.1 ). We denote by $M_{\nu}$ the sequence of $20 a^{\prime}$-operators corresponding to $A_{\nu}$ and $B_{\nu}$ and by $L_{\nu}$ the sequence of $N_{k}$-operators corresponding to $A_{\nu}$ and $B_{\nu}$ by tesseralization of $M_{\nu}$. We denote by $\left(f_{\nu}\right)$ the sequence obtained from $\left(F_{\nu}\right)$ by $(11)$ and (16). Now, we consider the initial-value problem $\left(P_{\nu}^{\prime}\right)_{20 a^{\prime}}$ : to find $\left(u_{\nu, n}\right)_{1 \leq n \leq 20 a^{\prime}}$ such that:

$$
M_{\nu} u_{\nu}=f_{\nu}, \quad u_{\nu, n}(0, x)=0, \quad 1 \leq n \leq 20 a^{\prime} .
$$

The Cauchy-Kowalevsky theorem applies; for each $\nu$, there exists a unique solution $u_{\nu}(t, x)$ which is real-analytic on all $\mathbb{R} \times \Omega$. Therefore, we obtain that $\left(u_{\nu, n}\right)_{1 \leq n \leq N_{k}}$ defined from $\left(u_{\nu, n}\right)_{1 \leq n \leq 4 a^{\prime}}$ by (10) is a solution of the tesseralized problem $\left(P_{\nu}^{\prime}\right)_{20 a^{\prime}}$ to the rank $N_{k}$ :

$$
L_{\nu} u_{\nu}=f_{\nu}, \quad u_{\nu, n}(0, x)=0,1 \leq n \leq 4 a^{\prime} .
$$

Now, since the tesseralized approximated problems have a solution, we can use the energy estimate (30) for $L_{\nu}$ instead of $L$, and so with a factor 2 for instance in the right member, denoting by $\mathcal{A}_{\nu}$ the approximation of $\mathcal{A}$ :

$$
\left(\int_{\mathcal{O}}\left|u_{\nu}\right|^{2} d V\right)^{\frac{1}{2}} \leq
$$

$$
\begin{aligned}
& k_{0} \sqrt{T}\left(\frac{2}{3}+\left\|\mathcal{A}_{\nu}\right\|_{4 a^{\prime} H^{k+2}(\mathcal{O})}^{4}+\|\chi\|_{4 a^{\prime} H^{k+2}(\mathcal{O})}^{4}\right)\left(\int_{\mathcal{O}}\left|L_{\nu}\left(u_{\nu}\right)\right|^{2} d V\right)^{\frac{1}{2}} \\
& \left(\int_{\mathcal{O}}\left|u_{\nu}\right|^{2} d V\right)^{\frac{1}{2}} \leq
\end{aligned}
$$$$
k_{0} \sqrt{T}\left(\frac{2}{3}+\left\|\mathcal{A}_{\nu}\right\|_{4 a^{\prime} H^{k+2}(\mathcal{O})}^{4}+\|\chi\|_{4 a^{\prime} H^{k+2}(\mathcal{O})}^{4}\right)\left(\int_{\mathcal{O}}\left|f_{\nu}\right|^{2} d V\right)^{\frac{1}{2}}
$$$$
\left(\int_{\mathcal{O}}\left|f_{\nu}\right|^{2} d V\right)^{\frac{1}{2}} \leq
$$$$
k_{0} \sqrt{T}\left(1+\|\mathcal{A}\|_{4 a^{\prime} H^{k+2}(\mathcal{O})}^{4}+\|\chi\|_{4 a^{\prime} H^{k+2}(\mathcal{O})}^{4}\right)\left(\int_{\mathcal{O}}|f|^{2} d V\right)^{\frac{1}{2}}
$$ 
we obtain that the sequence $\left(u_{\nu}\right)$ is bounded in ${ }^{N_{k}} L^{2}(] 0, T[\times \Omega)$ and that there exists a subsequence $u_{\nu_{j}} \rightarrow u$ weakly in ${ }^{N_{k}} L^{2}(] 0, T[\times \Omega)$. Therefore:

$$
L u=f \text {. }
$$

Moreover, $u$ satisfies inequality (30) with a $k_{0}$ equal to 3 times the original one and 1 instead of $\frac{1}{3}$. Back to problem $(P)$, if $F \in{ }^{4 a^{\prime}} H_{1}^{n+6}(] 0, T[\times \Omega)=F_{n}($ for $n \geq 0)$, we get:

$$
\partial_{0}^{m} F_{\nu a}(0, x)=0, \quad m=0,1 .
$$

Now, we consider the gauge field $A$ build on $u$ by relations (10). Then, $A$ is solution of the main equation of the (P) problem, with, according to (30), $A \in$ ${ }^{4 a^{\prime}} H^{n+7}(] 0, T[\times \Omega)$. Now, we have to show that $A$ satisfies the boundary conditions of:

$$
E_{n+1}={ }^{4 a^{\prime}} H_{3}^{n+7}(] 0, T[\times \Omega) .
$$

We denote by $B_{\nu a}(u)$ the line of $B u$ associated with the rank " $\nu, a$ " that is of rank $4 a^{\prime}+\nu+4(a-1)+1$. We get:

$$
\partial_{0}^{m} A_{\nu a}(0, x)=0, \quad \text { for } m=0,1
$$

and since $B_{\nu a}(u)$ is a linear combination of $\partial_{0}^{m} A_{\nu a}(0, x)$ for $m=0,1$, we get too:

$$
\partial_{0}^{2} A_{\nu a}(0, x)=F_{\nu a}(0, x)-\left(B_{\nu a}(u)\right)(0, x)=0
$$

and since $\partial_{0} B_{\nu a}(u)$ is a linear combination of $\partial_{0}^{m} A_{\nu a}(0, x)$ for $m=0,1,2$, we get:

$$
\partial_{0}^{3} A_{\nu a}(0, x)=\partial_{0} F_{\nu a}(0, x)-\partial_{0}\left(B_{\nu a}(u)\right)(0, x)=0 .
$$

Therefore:

$$
\partial_{0}^{\mu} A_{\nu a}(0, x)=0, \quad 0 \leq \mu, \nu \leq 3, \quad 1 \leq a \leq a^{\prime} .
$$

So, $A \in{ }^{4 a^{\prime}} H_{3}^{n+7}(] 0, T[\times \Omega)$. This $A$ is the solution of problem $(P)$, and the only one according to (30) itself. So, assumption 4 is fulfilled and a little bit more since, in (31), $\mathcal{A}$ can be chosen not only as a polynomial in $A_{n}$ but also as a distribution in $F_{n}$.

3.2.7. Assumption 6. We consider the $C$ in (9) and change (9) into:

$$
\begin{aligned}
& \left\|d Y M_{w \mathcal{A}}-d Y M_{w \mathcal{A}^{\prime}}\right\|_{L\left(4 a^{\prime} H_{3}^{n+2}(] 0, T[\times \Omega),{ }^{4 a^{\prime}} H_{1}^{n}(] 0, T[\times \Omega)\right)} \leq \\
& \max \left(C, C\|\chi\|_{4 a^{\prime} H_{3}^{n+2}(] 0, T[\times \Omega)}\right) \times\left\|\mathcal{A}-\mathcal{A}^{\prime}\right\|_{4 a^{\prime} H_{3}^{n+2}(] 0, T[\times \Omega)} \\
& \times\left(1+\|\mathcal{A}\|_{4 a^{\prime} H_{3}^{n+2}(] 0, T[\times \Omega)}+\left\|\mathcal{A}^{\prime}\right\|_{4 a^{\prime} H_{3}^{n+2}(] 0, T[\times \Omega)}\right)
\end{aligned}
$$

and the $k_{0}$ in (30). The product:

$$
12 \times \max \left(C, C\|\chi\|_{4 a^{\prime} H_{3}^{n+2}(] 0, T[\times \Omega)}\right) \times k_{0} \sqrt{T} \times\left(\|\chi\|_{4 a^{\prime} H^{k+2}(\mathcal{O})}^{4}+1\right)
$$

can be chosen $\geq 1$. So, assumption 6 is fulfilled.

3.2.8. Assumption \%. We notice that a minoration of $Y M_{w}(A)-Y M_{w}(B)$ can be done in the same way as the minoration of $\left\|d Y M_{w \mathcal{A}}^{-1} A\right\|_{4 a^{\prime} H^{k-1}(] 0, T[\times \Omega)}$ in (30), that is, by a rough majoration for $n \geq 0$ :

$$
\begin{aligned}
& \|A-B\|_{4 a^{\prime} H^{n+7}(] 0, T[\times \Omega)} \leq\left(\|A\|_{4 a^{\prime} H^{n+9}(] 0, T[\times \Omega)}^{4}+\|B\|_{4 a^{\prime} H^{n+9}(] 0, T[\times \Omega)}^{4}\right. \\
& +1) \times C_{n+6} \times\left\|Y M_{w}(A)-Y M_{w}(B)\right\|_{4 a^{\prime} H^{n+6}(] 0, T[\times \Omega)},
\end{aligned}
$$

where $C_{n+6}$ depends on $T$ and $n$ (this point here is without importance). So, assumption 7 is fulfilled. 
3.2.9. Assumption 8. We consider the sets $B_{n}$ of polynomials $P$ in $x_{0}, x_{1}, x_{2}$ and $x_{3}$ of degrees $\leq n$ with:

$$
\partial_{0}^{i} P\left(0, x_{1}, x_{2}, x_{3}\right)=0, \quad i=0,1 .
$$

So $B_{n}=C_{n}$. Therefore, $x \rightarrow f(x)-f(0)=Y M_{w}(x)-Y M_{w}(0)$ transforms any polynomial of $A_{n}$ in a polynomial which belongs to $B_{3 n}$. So, assumption 8 is fulfilled too. Hypotheses $8 \mathrm{a}$ ) and b) are evidently fulfilled. So, the whole assumptions of Theorem 2.1 are fulfilled.

Now, we still consider a constant gauge field $\chi$, any integer $m \geq 0$, any $\mathcal{A}_{\mu a} \in$ $A_{m}$, that is a gauge polynomial of degree $\leq m$ with:

$$
\partial_{0}^{k} \mathcal{A}_{\mu a}(0, x)=0, \quad 0 \leq k \leq 3,
$$

and any $r_{m}$ with:

$$
r_{m}<\frac{1}{4\left(\alpha_{m}\left\|\mathcal{A}_{\mu a}\right\|_{4 a^{\prime} H^{m}(] 0 ; T[\times \Omega)}^{4}+\beta_{m}\right) \gamma\left(2\left\|\mathcal{A}_{\mu a}\right\|_{4 a^{\prime} H^{m}(] 0 ; T[\times \Omega)}+3\right)}
$$

with $\gamma=\max \left(C, C\|\chi\|_{4 a^{\prime} H_{3}^{n+2}(] 0 ; T[\times \Omega)}\right)$ of paragraph 3.2.7, with the $\alpha_{m}=k_{0} \sqrt{T}$ and:

$$
\beta_{m}=k_{0} \sqrt{T}\|\chi\|_{4 a^{\prime} H^{0}(\mathcal{O})}^{4}+k_{0} \sqrt{T} .
$$

For any $F$ in ${ }^{4 a^{\prime}} H_{1}^{n+6}(] 0, T[\times \Omega)$ (still with $n \geq 0$ and with:

$$
\left\|F-Y M_{w}\left(\mathcal{A}_{\mu a}\right)\right\|_{4 a^{\prime} H_{1}^{n+6}(] 0, T[\times \Omega)} \leq \frac{r_{m}}{\alpha_{m}\left\|\mathcal{A}_{\mu a}\right\|_{4 a^{\prime} H_{3}^{m}(] 0, T[\times \Omega)}^{4}+\beta_{m}},
$$

there exists $A$ in ${ }^{4 a^{\prime}} H_{3}^{n+7}(] 0, T[\times \Omega)$ (still with $\left.n \geq 0\right)$ and with:

$$
\|A-\mathcal{A}\|_{4 a^{\prime} H_{3}^{n+7}(] 0, T[\times \Omega)} \leq 2 r_{m}
$$

such that:

$$
d Y M_{w} A=F .
$$

Moreover, according to the proof of Theorem 2.1, if $F$ is in a $C_{M}$ for a $M \geq 0$, then $A \in{ }^{4 a^{\prime}} H_{3}^{Q}(] 0, T[\times \Omega)$ for any $Q \geq 0$ so that $A \in{ }^{4 a^{\prime}} C_{3}^{\infty}(] 0, T[\times \Omega)$ (so that:

$$
\left.\partial_{0}^{i} A\left(0, x_{1}, x_{2}, x_{3}\right)=0, \quad 0 \leq i \leq 3\right) .
$$

Now, to show the existence of an infinity of non zero Yang-Mills fields, we consider the polynomial in $t$ quantum field ${ }_{N} \mathcal{A}_{\mu a}$ given by:

$$
\text { (32) } \quad{ }_{N} \mathcal{A}_{\mu a}(t, x)=k_{\mu a} T^{-N-0,7} t^{N}, \quad N \geq 4 .
$$

We can choose the $k_{\mu a}$ complex coefficients to get for $T$ sufficiently great and any $n \geq N-1$ and $n \geq 6$ :

$$
\begin{aligned}
& \left\|{ }_{N} \mathcal{A}_{\mu a}\right\|_{4 a^{\prime} H^{n+1}(] 0, T[\times \Omega)}=K_{0}^{\prime} T^{-N-0,7} \times \frac{T^{N+\frac{1}{2}}}{\sqrt{2 N+1}} \\
& \frac{K_{0}}{2 \sqrt{2 N+1}} T^{-0,2} \leq\left\|{ }_{N} \mathcal{A}_{\mu a}\right\|_{4 a^{\prime} H^{n+1}(] 0, T[\times \Omega)} \leq \frac{K_{0}}{\sqrt{2 N+1}} T^{-0,2},
\end{aligned}
$$

with a suitable constant $K_{0} \neq 0$, which can be chosen independent of $N$ when $N$ is sufficiently great. Still for $T$ sufficiently great, for $\chi$ sufficiently small and any 
$n \geq N:$

$$
\begin{aligned}
& \left\|Y M_{w}\left({ }_{N} \mathcal{A}_{\mu a}\right)\right\|_{4 a^{\prime}} H^{n}(] 0, T[\times \Omega) \\
& +k_{1}^{\prime} \times T^{-2 N-1,4} \times \frac{T^{\frac{4 N-1}{2}} \times N}{\sqrt{4 N-1}}+k_{2}^{\prime} \times T^{-3 N-0,7} \times \frac{T^{\frac{2(N-2)+1}{2}}}{\sqrt{2 N-3}} \times N(N-1) \\
& \leq k_{3}^{\prime} \frac{T^{-1,6}}{\sqrt{6 N+1}}
\end{aligned}
$$

for suitable constants $k_{0}^{\prime}, k_{1}^{\prime}, k_{2}^{\prime}$ and $k_{3}^{\prime}$. Now, still for $T$ sufficiently great, for $\chi$ sufficiently small, $r_{m}$ can be chosen such as to satisfy for $m \geq N$ :

$$
\begin{aligned}
& r_{m}< \\
& \frac{1}{4\left(\alpha_{m}\left\|_{N} \mathcal{A}_{\mu a}\right\|_{4 a^{\prime} H^{m}(] 0, T[\times \Omega)}^{4}+\beta_{m}\right) \gamma\left(2\left\|_{N} \mathcal{A}_{\mu a}\right\|_{4 a^{\prime} H^{m}(] 0, T[\times \Omega)}+3\right)} \\
& r_{m} \leq K_{1} T^{-\frac{1}{2}}
\end{aligned}
$$

and:

$$
\frac{r_{m}}{\alpha_{m}\left\|N \mathcal{A}_{\mu a}\right\|_{4 a^{\prime} H^{m}(] 0, T[\times \Omega)}^{4}+\beta_{m}} \geq K_{2} T^{-1}
$$

with suitable constants $K_{1}$ and $K_{2}$ depending on $N$. Now, for $T$ sufficiently great and $\chi$ taken sufficiently small, we get for any $n \geq N-1$ and $n \geq 6$ and $m \geq N$ :

$$
\left\|{ }_{N} \mathcal{A}_{\mu a}\right\|_{4 a^{\prime} H^{m}(] 0, T[\times \Omega)}>2 K_{1} T^{-\frac{1}{2}}>2 r_{m}
$$

and:

$$
\begin{aligned}
& \left\|Y M_{w}\left({ }_{N} \mathcal{A}_{\mu a}\right)\right\|_{4 a^{\prime} H^{m}(] 0, T[\times \Omega)}<K_{2} T^{-1} \\
& \left\|Y M_{w}\left({ }_{N} \mathcal{A}_{\mu a}\right)\right\|_{4 a^{\prime} H^{n}(] 0, T[\times \Omega)}<\frac{r_{m}}{\alpha_{m}\left\|{ }_{N} \mathcal{A}_{\mu a}\right\|_{4 a^{\prime} H^{m}(] 0, T[\times \Omega)}^{4}+\beta_{m}} .
\end{aligned}
$$

So, for $n \geq 6$ (since $\left.F_{0}={ }^{4 a^{\prime}} H_{1}^{6}(] 0, T[\times \Omega)\right)$, the trivial gauge field 0 is in:

$$
B_{4 a^{\prime} H_{1}^{n}(] 0, T[\times \Omega)}\left(Y M_{w}\left({ }_{N} \mathcal{A}_{\mu a}\right), \frac{r_{m}}{\alpha_{m}\left\|_{N} \mathcal{A}_{\mu a}\right\|_{4 a^{\prime} H^{m}(] 0, T[\times \Omega)}^{4}+\beta_{m}}\right)
$$

and there exists a non-trivial gauge field $A_{N}$ in $B_{4 a^{\prime} H^{n+3}(] 0, T[\times \Omega)}\left({ }_{N} \mathcal{A}_{\mu a}, 2 r_{m}\right)$, with:

$$
Y M_{w}\left(\chi+A_{N}\right)=0 .
$$

Therefore, $\chi+A_{N}=\left(\chi_{\nu a}+A_{N, \nu a}\right)_{0 \leq \nu \leq 3,1 \leq a \leq a^{\prime}}$ with $N \geq 4$ is a sequence of non trivial "w" Yang-Mills fields. So, making $T$ depend on $N$ and choosing $T_{N}$ as great as we want, we have build a sequence of non trivial Yang-mills fields, different each other, with $A_{N} \in{ }^{4 a^{\prime}} C_{3}^{\infty}\left(\left[0, T_{N}\right] \times \Omega\right)$ and by restriction in ${ }^{4 a^{\prime}} C_{3}^{\infty}([0, T] \times \Omega)$ with $T=T_{4}$. So $\chi+A_{N} \in{ }^{4 a^{\prime}} C^{\infty}([0, T] \times \Omega)$. By choosing a non countable infinity of constant non-trivial fields $\chi$, since $\left(A_{N}+\chi\right)(0, x)=\chi$, we obtain a non countable infinity of sequences of Yang-Mills fields.

Now, we have to show that these quantum fields are not only "w" Yang-Mills but Landau-Lorentz Yang-Mills ones, that is:

$$
\partial^{\mu}\left(\chi_{\mu a}+A_{N, \mu a}\right)=0, \quad 1 \leq a \leq a^{\prime}, \quad N \geq 4 .
$$

Now:

$$
D^{\mu} F_{\mu \nu}+\partial_{\nu} \partial^{\mu}\left(\chi_{\mu}+A_{N, \mu}\right)=0, \quad 0 \leq \nu \leq 3
$$


with the strength tensor $F_{\mu \nu}$ associated with $\chi+A_{N}$. Let's apply $D^{\nu}$ (with $D^{\nu}$ still given by (2)):

$$
D^{\nu} D^{\mu} F_{\mu \nu}+\partial^{\nu} \partial_{\nu} \partial^{\mu}\left(\chi_{\mu}+A_{N, \mu}\right)-g\left[\chi+A_{N}^{\nu}, \partial_{\nu} \partial^{\mu}\left(\chi+A_{N, \mu}\right)\right]=0 .
$$

Since:

we get that:

$$
D^{\nu} D^{\mu} F_{\mu \nu}=0,
$$

$$
\partial^{\nu} \partial_{\nu} \partial^{\mu} A_{N, \mu}-g \partial_{\nu}\left[\chi^{\nu}+A_{N}^{\nu}, \partial^{\mu} A_{N, \mu}\right]+g\left[\partial_{\nu} A_{N}^{\nu}, \partial^{\mu} A_{N, \mu}\right]=0 .
$$

Now:

$$
\left[\partial_{\nu} A_{N}^{\nu}, \partial^{\mu} A_{N, \mu}\right]=0
$$

affords that:

$$
\partial^{\nu} \partial_{\nu} \partial^{\mu} A_{N, \mu a}-g \partial_{\nu}\left[C_{a}^{b c}\left(\chi^{\nu b}+A_{N}^{\nu b}\right) \partial^{\mu} A_{N, \mu c}\right]=0, \quad 1 \leq a \leq a^{\prime} .
$$

Now, we introduce the following linear system:

$$
\partial^{\nu} \partial_{\nu} u_{a}-g \partial_{\nu}\left[C_{a}^{b c}\left(\chi^{\nu b}+A_{N}^{\nu b}\right) u_{c}\right]=0, \quad 1 \leq a \leq a^{\prime} .
$$

If:

$$
u_{n}=\partial_{0} u_{n}=0 \quad \text { on }\{0\} \times \Omega,
$$

(34) has 0 for unique solution. Since $A \in{ }^{4 a^{\prime}} C_{3}^{\infty}([0, T] \times \Omega)$ :

$$
\partial^{\mu} A_{N, \mu a}(0, x)=\partial_{0} \partial_{N, \mu a}^{\mu}(0, x)=0,
$$

so that (33) occurs and $\chi=A_{N}$ is a smooth Landau-Lorentz Yang-Mills field.

We consider an increasing sequence $T_{n}$ with:

$$
\lim _{n \rightarrow+\infty} T_{n}=+\infty \text {. }
$$

Then, it is possible to obtain smooth Yang-Mills fields. Indeed, we cover $\mathbb{R}_{t}^{+} \times \mathbb{R}^{3}$ with "pieces":

$$
\left.M_{n}=\right] 0, T_{n}\left[\times\left\{\left(x_{2}, x_{3}, x_{4}\right), \sqrt{x_{2}^{2}+x_{3}^{2}+x_{4}^{2}}<n\right\}\right.
$$

in whole $\mathbb{R}_{t}^{+} \times \mathbb{R}^{3}$. Considering a fixed ${ }_{N} \mathcal{A}$ as in (32), we obtain smooth Yang-Mills fields $A_{N, n}$ in $\overline{M_{n}}$. According to the bowls described above, since the rays $r_{m}$ decrease when $T$ grows, we get:

$$
A_{N, n^{\prime}} \equiv A_{N, n} \quad \text { for } n^{\prime} \geq n \text { in } \overline{M_{n}} .
$$

Indeed, let's denote by $r_{m_{T_{n}}}$ the rays of the bowls of Theorem $2.1 \mathrm{in:}$

$$
{ }^{4 a^{\prime}} H_{1}^{n}(] 0, T_{n}[\times \Omega) .
$$

If:

$$
A_{N, n^{\prime}} \in B_{4 a^{\prime} H_{1}^{n}(] 0, T_{n^{\prime}}[\times \Omega)}\left(Y M_{w}\left({ }_{N} \mathcal{A}_{\mu a}\right), \frac{r_{m_{T_{n^{\prime}}}}}{\alpha_{m}\left\|_{N} \mathcal{A}_{\mu a}\right\|_{4 a^{\prime} H^{m}(] 0, T_{n^{\prime}}[\times \Omega[)}^{4}+\beta_{m}}\right),
$$

then, since $r_{m_{T_{n^{\prime}}}}$ can be chosen $<r_{m_{T_{n}}}$ (even if $T_{n}$ has not to be modified by taking greater values):

$$
\begin{aligned}
& \left.A_{N, n^{\prime}}\right|_{\left[0, T_{n}\right]} \in \\
& B_{4 a^{\prime} H_{1}^{n}(] 0, T_{n}[\times \Omega)}\left(Y M_{w}\left({ }_{N} \mathcal{A}_{\mu a}\right), \frac{r_{m_{T_{n}}}}{\alpha_{m}\left\|\mathcal{A}_{\mu a}\right\|_{4 a^{\prime} H^{m}(] 0, T_{n}[\times \Omega[)}^{4}+\beta_{m}}\right) .
\end{aligned}
$$


So $\lim _{n \rightarrow+\infty} A_{N, n}$ is a smooth Landau-Lorentz gauge field in $\mathbb{R}_{t}^{+} \times \mathbb{R}^{3}$. Now, we can imagine:

$$
\left.M_{n}^{\prime}=\right]-n, T_{n}\left[\times\left\{\left(x_{2}, x_{3}, x_{4}\right), \sqrt{x_{2}^{2}+x_{3}^{2}+x_{4}^{2}}<n\right\} .\right.
$$

We can consider a smooth prolongment ${ }_{N}^{n} \mathcal{A}$ of ${ }_{N} \mathcal{A}$ (as in (32)) still with an increasing sequence $T_{n}$ with:

$$
\lim _{n \rightarrow+\infty} T_{n}=+\infty
$$

and such that:

$$
\partial_{0 N}^{i n} \mathcal{A}_{\mu a}(-n, x)=0 \quad \text { for } 0 \leq i \leq 3 .
$$

Then, we obtain smooth Yang-Mills fields $A_{N, n}$ in $\overline{M_{n}^{\prime}}$ such that:

$$
A_{N, m} \equiv A_{N, n} \quad \text { for } n \geq m \text { in } \overline{M_{n}} .
$$

So $\lim _{n \rightarrow+\infty} A_{N, n}$ is a smooth Landau-Lorentz gauge field in whole $\mathbb{R}^{4}$.

\section{Strength tensors of a certain kind of super-symmetry type}

4.1. Motion equation. We consider a product of a finite number of Lie algebras $L_{1}, \ldots, L_{A^{\prime}}$; the generators of $L_{K}$ are denoted by $\left\{t_{a K}\right\}_{1 \leq a \leq t_{K}}$. We consider a partition $\left\{\mathcal{L}_{I}\right\}_{1 \leq I \leq \mathcal{I}}$ of the set $\left\{L_{K}\right\}_{1 \leq K \leq A^{\prime}}$ with:

$$
\mathcal{L}_{I}=\left\{L_{K}\right\}_{K \in \mathcal{I}_{I}} \text {. }
$$

We suppose that:

1) for any $I$ with $1 \leq I \leq \mathcal{I}$ :

$$
\forall E, F \in \mathcal{I}_{I}, \quad \forall a, b \text { with } 1 \leq a \leq t_{E}, 1 \leq b \leq t_{F}, \quad \lambda t_{a E}+\mu t_{b F}
$$

brings an element of a complex vectorial space $M_{I}$ containing $\mathcal{L}_{I}$, so that $\lambda=\mu=0$ implies $\lambda t_{a E}+\mu t_{b F}=0$ in $M_{I}$,

2 ) there exists a product " $t_{a E} t_{b F}$ " (non necessarily commutative), inside $\mathcal{L}_{I}$ with $1 \leq I \leq \mathcal{I}$ :

$$
t_{a e} t_{b F}=\sum_{\substack{\kappa \in \mathcal{I}_{I} \\ 1 \leq c \leq t_{K}}} \nu_{a E b F c K} t_{c K}
$$

with some complex coefficients $\nu_{a E b F c K}$.

We consider the gauge fields $\left(V_{K}^{\mu}(x)=V_{K a}^{\mu}(x) t_{K}^{a}=\left(\chi_{K a}^{\mu}+W_{K a}^{\mu}\right) t_{K}^{a}\right)$ with $0 \leq \mu \leq 3$ and $1 \leq K \leq A^{\prime}$ with a constant field $\chi_{K a}^{\mu}$. We consider the strength tensor $F_{K \mu \nu}$ with $K \in \mathcal{I}_{I}, 0 \leq \mu, \nu \leq 3$ defined by:

$$
F_{K \mu \nu}=\partial_{\mu} V_{K \nu}-\partial_{\nu} V_{K \mu}+\sum_{M, N \in \mathcal{I}_{I}} R_{K M N} V_{M \mu} V_{N \nu}+\sum_{M, N \in \mathcal{I}_{I}} L_{K M N} V_{M \nu} V_{N \mu},
$$

where the $R_{K M N}$ and $L_{K M N}$ can be polynomials (and not numbers any more). We notice that the last sum in $F_{K \mu \nu}$ is not considered in classical super-symmetry theories and that the $R_{K M N}$ are only complex numbers in those theories (see Weinberg $[6$, volume 3,page 128,(27.3.2)]).

So, the present paragraph 4 will generalize paragraph 3 with its commutators.

Then, even if the Yang-Mills equation of the motion of the gauge field $V$ is not very clear in the books we've read, we can imagine that this equation features in 
the following form:

$$
\begin{aligned}
& \forall I, \quad 1 \leq I \leq \mathcal{I}, \quad \forall K \in \mathcal{I}_{I}, \quad \nu=0,1,2,3 \\
& (35) \quad \partial^{\mu} \sum_{K \in \mathcal{I}_{I}} F_{K \mu \nu}+\sum_{K \in \mathcal{I}_{I}} \sum_{M, N \in \mathcal{I}_{I}} R_{K M N} V_{M}^{\mu} F_{N \mu \nu} \\
& +\sum_{K \in \mathcal{I}_{I}} \sum_{M, N \in \mathcal{I}_{I}} L_{K M N} F_{N \nu}^{\mu} V_{M \mu}=0
\end{aligned}
$$

We denote (35) by:

$$
S Y M(V)=0 .
$$

$S Y M$ is written for "super-symmetric Yang-Mills". So, we get (35) if:

$$
\left(\left(\partial^{\mu} \partial_{\mu} V_{K \nu a}-\partial_{\nu} \partial^{\mu} V_{K \mu a}+E_{a K \nu}(V)\right) t_{K}^{a}=0\right)_{\substack{1 \leq K \leq A^{\prime} \\ 0 \leq \nu \leq 3}}
$$

with:

$$
\begin{aligned}
& E_{a K \nu}(V)=\sum_{\substack{M, N, O \in \mathcal{I}_{I} \\
1 \leq b \leq t_{M} \\
1 \leq c \leq t_{N} \\
1 \leq d \leq t_{O}}} C_{K \nu a M N O b c d} V_{M b}^{\mu} V_{N \mu c} V_{O \nu d} \\
& +\sum_{\substack{M, N \in \mathcal{I}_{I} \\
1 \leq b \leq \pm_{M} \\
1 \leq c \leq t_{N}}} D_{K \nu a M N b c} V_{M b}^{\mu}\left(\partial_{\mu} V_{N \nu c}\right)+\sum_{\substack{M, N \in \mathcal{I}_{I} \\
1 \leq b \leq t_{M} \\
1 \leq c \leq t_{N}}} E_{K \nu a M N b c} V_{M b}^{\mu}\left(\partial_{\nu} V_{N \nu c}\right) \\
& +\sum_{\substack{M, N \in \mathcal{I}_{I} \\
1 \leq b \leq t_{M} \\
1 \leq c \leq t_{N}}} G_{K \nu a M N b c} V_{M \nu b} \partial^{\mu} V_{N \mu c}+\sum_{\substack{M, N \in \mathcal{I}_{I} \\
1 \leq b \leq t_{M} \\
1 \leq c \leq t_{N}}} H_{K \nu a M N b c}^{\mu} V_{M \mu b} V_{N \nu c} .
\end{aligned}
$$

$C_{K \nu a M N O b c d}, D_{K \nu a M N b c}, E_{K \nu a M N b c}, G_{K \nu a M N b c}$ and $H_{K \mu \nu a M N b c}$ are polynomials expressible by the $R_{K M N}$ and $L_{K M N}$ ones.

Now, (36) occurs if for $1 \leq K \leq A^{\prime}, 1 \leq a \leq t_{K}, 0 \leq \nu \leq 3$ :

$$
\partial^{\mu} \partial_{\mu} V_{K \nu a}-\partial_{\nu} \partial^{\mu} V_{K \mu a}+E_{a K \nu}(V)=0 .
$$

4.2. Finite propagation speed. Now, we consider only:

$$
\partial^{\mu} \partial_{\mu} V_{K \nu a}+E_{a K \nu}(V)=0
$$

still for $1 \leq K \leq A^{\prime}, 1 \leq a \leq t_{K}, 0 \leq \nu \leq 3$. We denote by $S Y M_{w} W_{K \nu a}$ (recall that $V=\chi+W)$ the left member of (39). We put:

$$
z=4 \sum_{k=1}^{A^{\prime}} t_{k} .
$$

Now, like in paragraph 3.2, we consider the indexation $k$ of the Sobolev spaces such that $S Y M$ acts from ${ }^{z} H_{3}^{k+8}(] 0, T[\times \Omega)$ into ${ }^{z} H_{1}^{k+6}(] 0, T[\times \Omega)$ for $k \geq 0$. But, (39) is of the same type as (6) and can be treated by Theorem 2.1, exactly in the same way. So, there exists a non countable infinity of sequences of smooth gauge fields $V_{N}$ different each other with the same properties as described in paragraph 3.2.9, solution to (39).

Now, we just have to show that:

$$
\partial^{\mu} V_{K \mu a}=0, \quad 1 \leq K \leq A^{\prime}, \quad 1 \leq a \leq t_{K}
$$


to obtain a Landau-Lorentz gauge field solution to the Yang-Mills equation (38). We apply $\partial^{\nu}$ to (39) and we suppose that we obtain:

$$
\partial^{\mu} \partial_{\mu} \partial^{\nu} V_{K \nu a}+g_{K a}^{L_{b}} \partial^{\mu} V_{L \mu b}+h_{K a}^{L_{\mu b}} V_{L \mu b}=0, \quad 1 \leq K \leq A^{\prime}, 1 \leq a \leq t_{K},
$$

where $g_{K a}^{L_{b}}$ and $h_{K a}^{L_{\mu b}}$ have the same regularity as $V, V^{2}, \partial V, V \partial V$ or $\partial^{2} V$. Now, we consider the following sequence:

$$
u_{B<K} t_{B}+a+1=\partial^{\nu} V_{K \nu a} .
$$

We put:

$$
z^{\prime}=\sum_{K=1}^{A^{\prime}} t_{K}
$$

If $z^{\prime}<n \leq 5 z^{\prime}$ we put:

$$
n=z^{\prime}+n_{0} \times z^{\prime}+n_{1}
$$

with:

$$
\left\{\begin{array}{l}
0 \leq n_{0} \leq 3 \\
0 \leq n_{1} \leq z^{\prime}
\end{array}\right.
$$

For $z^{\prime}<n \leq 5 z^{\prime}$, we put:

$$
u_{n}=\partial_{n_{0}} u_{n_{1}}
$$

We consider the other sequence:

$$
\left\{\begin{array}{l}
v_{n}=0 \quad 1 \leq n \leq z^{\prime} \\
v_{z^{\prime}+} \sum_{B<K} t_{B}+a+1=g_{K a}^{L_{b}} u \sum_{B<L} t_{B}+b+1 \\
\text { for } 1 \leq K \leq h_{K a}^{L_{\mu b}} V_{L \mu b}, 1 \leq a \leq t_{K} \\
v_{n}=0 \quad 2 z^{\prime}<n \leq 5 z^{\prime}
\end{array} .\right.
$$

Then, we consider the matrices $A_{1}, A_{2}, A_{3}$ defined by:

$$
\begin{aligned}
A_{1} & =\left(\begin{array}{lllll}
0_{z^{\prime}} & 0_{z^{\prime}} & 0_{z^{\prime}} & 0_{z^{\prime}} & 0_{z^{\prime}} \\
0_{z^{\prime}} & 0_{z^{\prime}} & -I d_{z^{\prime}} & 0_{z^{\prime}} & 0_{z^{\prime}} \\
0_{z^{\prime}} & -I d_{z^{\prime}} & 0_{z^{\prime}} & 0_{z^{\prime}} & 0_{z^{\prime}} \\
0_{z^{\prime}} & 0_{z^{\prime}} & 0_{z^{\prime}} & 0_{z^{\prime}} & 0_{z^{\prime}} \\
0_{z^{\prime}} & 0_{z^{\prime}} & 0_{z^{\prime}} & 0_{z^{\prime}} & 0_{z^{\prime}}
\end{array}\right), \\
A_{2} & =\left(\begin{array}{lllll}
00_{z^{\prime}} & 0_{z^{\prime}} & 0_{z^{\prime}} & 0_{z^{\prime}} & 0_{z^{\prime}} \\
0_{z^{\prime}} & 0_{z^{\prime}} & 0_{z^{\prime}} & -I d_{z^{\prime}} & 0_{z^{\prime}} \\
0_{z^{\prime}} & 0_{z^{\prime}} & 0_{z^{\prime}} & 0_{z^{\prime}} & 0_{z^{\prime}} \\
0_{z^{\prime}} & -I d_{z^{\prime}} & 0_{z^{\prime}} & 0_{z^{\prime}} & 0_{z^{\prime}} \\
0_{z^{\prime}} & 0_{z^{\prime}} & 0_{z^{\prime}} & 0_{z^{\prime}} & 0_{z^{\prime}}
\end{array}\right), \\
A_{2} & =\left(\begin{array}{lllll}
0_{z^{\prime}} & 0_{z^{\prime}} & 0_{z^{\prime}} & 0_{z^{\prime}} & 0_{z^{\prime}} \\
0_{z^{\prime}} & 0_{z^{\prime}} & 0_{z^{\prime}} & 0_{z^{\prime}} & -I d_{z^{\prime}} \\
0_{z^{\prime}} & 0_{z^{\prime}} & 0_{z^{\prime}} & 0_{z^{\prime}} & 0_{z^{\prime}} \\
0_{z^{\prime}} & 0_{z^{\prime}} & 0_{z^{\prime}} & 0_{z^{\prime}} & 0_{z^{\prime}} \\
0_{z^{\prime}} & -I d_{z^{\prime}} & 0_{z^{\prime}} & 0_{z^{\prime}} & 0_{z^{\prime}}
\end{array}\right) .
\end{aligned}
$$

So, the system (41) can be written:

$$
L u=\partial_{0} u+\sum_{j=1}^{3} A_{j} \partial_{j} u+B u+v=0,
$$


where the $B$ matrix is the $5 z^{\prime} \times 5 z^{\prime}$ matrix:

$$
B=\left(\begin{array}{ccccc}
0_{z^{\prime}} & -I d_{z^{\prime}} & 0_{z^{\prime}} & 0_{z^{\prime}} & 0_{z^{\prime}} \\
0_{z^{\prime}} & 0_{z^{\prime}} & 0_{z^{\prime}} & 0_{z^{\prime}} & 0_{z^{\prime}} \\
0_{z^{\prime}} & 0_{z^{\prime}} & 0_{z^{\prime}} & 0_{z^{\prime}} & 0_{z^{\prime}} \\
0_{z^{\prime}} & 0_{z^{\prime}} & 0_{z^{\prime}} & 0_{z^{\prime}} & 0_{z^{\prime}} \\
0_{z^{\prime}} & 0_{z^{\prime}} & 0_{z^{\prime}} & 0_{z^{\prime}} & 0_{z^{\prime}}
\end{array}\right)
$$

and where $v$ is the $5 z^{\prime} \times 1$ column matrix:

$$
v=\left(\begin{array}{c}
0_{z^{\prime}}^{\prime} \\
v_{1} \\
v_{2} \\
\cdots \\
v_{z^{\prime}} \\
0_{z^{\prime}}^{\prime} \\
0_{z^{\prime}}^{\prime} \\
0_{z^{\prime}}^{\prime}
\end{array}\right)
$$

where $0_{z^{\prime}}^{\prime}$ is a $z^{\prime}$-vertical matrix only composed by $0 \mathrm{~s}$.

4.3. Finite propagation speed. Then, we use the same notation as in paragraph 3.2.5 with $5 z^{\prime}$ instead of $N_{k}$ : since $V \in{ }^{z^{\prime}} H_{3}^{k+6}$, we get that $u_{n}(0, x)=0$ for $x \in \Omega$ and $1 \leq n \leq z^{\prime}$. Moreover:

$$
\partial_{j} u_{n}(0, x)=0, \quad \text { for } 1 \leq n \leq z^{\prime}, \quad 0 \leq j \leq 3,
$$

so that $u_{n}(0, x)=0$ for any $n$ and:

$$
\begin{aligned}
(L u, u)_{s}+(u, L u)_{s}-(u, B u)_{s}-(u, v)_{s}-(B u, u)_{s}-(v, u)_{s}= & \int_{\Sigma_{s}}|u|^{2} d S \\
& -\int_{\Sigma_{0}}|u|^{2} d S \\
(43) \quad 2 \operatorname{Re}(L u, u)_{s}-2 \operatorname{Re}(B u, u)_{s}-2 \operatorname{Re}(v, u)_{s} & =\int_{\Sigma_{s}}|u|^{2} d S
\end{aligned}
$$

Since:

$$
V_{K \nu a}(0, x)=0,
$$

we get (according to Taylor [5,volume 1,(5.34),page 439]):

$$
\left\|V_{K \nu a}\right\|_{L^{2}(\mathcal{O}(s))} \leq C_{0}\left\|\partial^{\nu} V_{K \nu a}\right\|_{L^{2}(\mathcal{O}(s))} .
$$

Now, since $V \in{ }^{z^{\prime}} H_{3}^{k+7}$ with $k \geq 0$, the coefficients of $u$ and $V$ in the expression (42) of $v_{n}$ in function of $u$ and $V$ are $L^{\infty}$, so by (44), we obtain that:

$$
\|v\|_{z^{\prime} L^{2}(\mathcal{O}(s))} \leq C_{1}\|u\|_{z^{\prime} L^{2}(\mathcal{O}(s))} .
$$

Therefore, if we put:

$$
E(s)=\|u\|_{z^{\prime} L^{2}(\mathcal{O}(s))}^{2},
$$


according to (43) and (45), we obtain that:

$$
\begin{aligned}
& \frac{d E}{d s}(s) \leq 2 \int_{\mathcal{O}(s)}|L u \times \bar{u}| d V+2 \int_{\mathcal{O}(s)}|u|^{2} d V+2 C_{1} \int_{\mathcal{O}(s)}|v \times \bar{u}| d V \\
& \frac{d E}{d s}(s) \leq C_{3}\left(\|L u\|_{z^{\prime} L^{2}(\mathcal{O}(s))}^{2}+\|v\|_{z^{\prime} L^{2}(\mathcal{O}(s))}^{2}+\|u\|_{z^{\prime} L^{2}(\mathcal{O}(s))}^{2}\right) \\
& \frac{d E}{d s}(s) \leq C_{3}\|L u\|_{z^{\prime} L^{2}(\mathcal{O}(s))}^{2}+C_{4} E(s) .
\end{aligned}
$$

Then, if we apply the Gronwall lemma, for $s=T$ :

$$
\|u\|_{z^{\prime} L(\mathcal{O})}^{2} \leq C_{3}\|L u\|_{z^{\prime} L(\mathcal{O})}^{2} \times e^{C_{4} T} .
$$

So, since $L u=0$, we obtain $u=0$ and so:

$$
\partial^{\nu} V_{K \nu a}=0, \quad 1 \leq K \leq A^{\prime}, 1 \leq a \leq t_{K} .
$$

Therefore, we obtain that $V$ is a Landau-Lorentz field and so:

$$
\partial_{\nu} \partial^{\mu} V_{K \mu a}=0, \quad 1 \leq K \leq A^{\prime}, 1 \leq a \leq t_{K}, 0 \leq \nu \leq 3 .
$$

So, if we introduce the opposite of the left member of (46) in (39), we obtain the Yang-Mills equations (38). Therefore, relatively to the compact manifold with boundary $P$ of $\mathbb{R}^{3}$, we obtain that (35) occurs in $] 0, T[\times P$. So, we obtain a non countable infinity of sequences of non trivial super-symmetric fields under conditions (41).

Now, if we cover $\mathbb{R}^{4}$ with the same pieces $M_{i}$ as in paragraph 3.2 .9 , we get smooth super-symmetric Landau-Lorentz gauge fields in whole $\mathbb{R}^{4}$.

Moreover, like for the classical Yang-Mills fields, the question of the uniqueness of the Yang-Mills super-symmetric fields of the type considered here remains open.

\section{Conclusion}

Theorem 2.1 can be applied in a rather easy way in some questions of existence (and uniqueness) of the solution to nonlinear partial differential problems; among various famous problems, we first think to nonlinear hyperbolic equations, but also to nonlinear parabolic problems that can't be treated by the Hadamard-Lévy theorem itself. Among nonlinear parabolic equations, we especially think to the nonlinear Schrödinger's one that can be treated or by the means of the present paper or on the basis of Lions-Magenes [4,volume 2,chapitre 5]; the roots of the Lions-Magenes analytic method are to be found in the coercivity inequalities of the Faedo-Galerkine method.

Concerning the Yang-Mills equation itself, the question of the existence of a solution to the non homogeneous one $(Y M u=f$ with $f \neq 0)$ remains open but can be treated by the present paper for some particular classes of fields $f$. Still about Yang-Mills theory, we think that it is possible to prove the existence of Yang-Mills Coulomb gauge fields. Since Einstein's equation and other ones in the theory of relativity look like the Yang-Mills one, we don't know how far the present researches could be applied to relativity.

Now, it would be interesting to apply the methods of this paper to any Riemannian metric instead of the simple Lorentz metric. Moreover, an important problem should be a control theory for the Yang-Mills equation.

The main question is the existence or not of a mass gap to know if the passage from the vacuum to the matter can be continuous. 
In a higher point of view, we think that some classical theorems of local analysis (Taylor expansions etc.) can be generalized either in the surround of the metric spaces or of the Banach spaces.

\section{References}

[1] A. Avez, Calcul différentiel, Masson, Paris, New York, Barcelone, Milan, Mexico, São Paulo, 1983

[2] T. Frankel

The Geometry of Physics, An Introcution, (first edition 1997), Cambridge University Press, Cambridge GB, New York, Melbourne, Madrid, 1999

[3] C. Itzykson \& J.-B. Zuber,

Quantum Field Theory, (first edition 1980), McGraw-Hill International Editions, New York, St Louis, San Francisco, Auckland, Bogota, Hamburg, London, Madrid, Mexico, Montreal, New Delhi, Panama, Paris, São Paulo,Singapore, Sydney, Tokyo, Toronto, 1985

[4] J.L. Lions and E. Magenes

Problèmes aux limites non homogènes, 3 volumes, Dunod, Paris, 1968

[5] M.E. Taylor

Partial Differential Equations, 3 volumes, Springer, 117, New York, Berlin, Heidelberg, Barcelona, Budapest, Hong Kong, London, Milan, Paris, Santa Clara, Singapore, 1997

[6] S. Weinberg

The Quantum Theory of Fields, 3 volumes, Cambridge University Press, Cambridge GB, New York, Melbourne, Madrid ; volume 1, 1999 (first edition 1995); volume 2, 2000 (first edition 1996); volume 3, 2000.

Institut Pierre Simon Laplace, 5, Rue du Lieutenant de Sesmaisons, 44300 Nantes, FRANCE

E-mail address: gib.pecot@infonie.fr 